MATHEMATICS OF COMPUTATION

Volume 74, Number 252, Pages 1895-1921

S 0025-5718(05)01742-4

Article electronically published on May 5, 2005

\title{
CONSTRUCTION ALGORITHMS FOR POLYNOMIAL LATTICE RULES FOR MULTIVARIATE INTEGRATION
}

\author{
J. DICK, F. Y. KUO, F. PILLICHSHAMMER, AND I. H. SLOAN
}

\begin{abstract}
We introduce a new construction algorithm for digital nets for integration in certain weighted tensor product Hilbert spaces. The first weighted Hilbert space we consider is based on Walsh functions. Dick and Pillichshammer calculated the worst-case error for integration using digital nets for this space. Here we extend this result to a special construction method for digital nets based on polynomials over finite fields. This result allows us to find polynomials which yield a small worst-case error by computer search. We prove an upper bound on the worst-case error for digital nets obtained by such a search algorithm which shows that the convergence rate is best possible and that strong tractability holds under some condition on the weights.

We extend the results for the weighted Hilbert space based on Walsh functions to weighted Sobolev spaces. In this case we use randomly digitally shifted digital nets. The construction principle is the same as before, only the worstcase error is slightly different. Again digital nets obtained from our search algorithm yield a worst-case error achieving the optimal rate of convergence and as before strong tractability holds under some condition on the weights. These results show that such a construction of digital nets yields the until now best known results of this kind and that our construction methods are comparable to the construction methods known for lattice rules.

We conclude the article with numerical results comparing the expected worst-case error for randomly digitally shifted digital nets with those for randomly shifted lattice rules.
\end{abstract}

\section{INTRODUCTION}

We are interested in the study of multivariate integration, more precisely, we want to approximate the $s$-dimensional integral $\int_{[0,1)^{s}} f(\boldsymbol{x}) \mathrm{d} \boldsymbol{x}$ by a quadrature rule. This is done by calculating the average of the values $f\left(\boldsymbol{x}_{h}\right)$ for a point set $\left\{\boldsymbol{x}_{0}, \ldots, \boldsymbol{x}_{n-1}\right\}$. For Monte Carlo rules the point set is chosen randomly, whereas for quasi-Monte Carlo (QMC) rules the point set is chosen deterministically with the aim of distributing the points as uniformly as possible in the unit cube.

Sobol' 34] introduced a construction method for well-distributed point sets in the unit cube based on the digits of the points. Later Faure [8] established another method to generate well-distributed points and subsequently Niederreiter [19] gave a detailed introduction and investigation of the general concept, which he called $(t, m, s)$-nets and $(t, s)$-sequences in base $b$. These are point sets in $[0,1)^{s}$, where a

Received by the editor January 9, 2004 and, in revised form, May 4, 2004.

2000 Mathematics Subject Classification. Primary 65C05, 65D30.

Key words and phrases. Quasi-Monte Carlo, numerical integration, polynomial lattice rules.

(C)2005 American Mathematical Society 
$(t, m, s)$-net consists of $b^{m}$ points and a $(t, s)$-sequence provides an infinite number of points. The $t$ in $(t, m, s)$ and $(t, s)$ is a non-negative integer indicating the distribution properties of the point set (hence $t$ is also called the quality parameter of the point set). A small $t$ corresponds to a point set that is well distributed.

In a series of papers Niederreiter established several powerful construction methods (see 23] for an overview). All those methods are based on a general construction principle which uses linear algebra over finite fields (or more generally over finite commutative rings). The resulting point sets from the general construction principle are called digital $(t, m, s)$-nets and digital $(t, s)$-sequences over a finite field. One of the construction methods formulated by Niederreiter uses polynomials over finite fields (see 21]). (Hence QMC rules using those point sets are called polynomial lattice rules; see [16.) Polynomials which yield well-distributed point sets for sequences are known (see [21, 34]) and have been used successfully in many applications. On the other hand finite point sets, that is $(t, m, s)$-nets, can have a significantly smaller quality parameter $t$. At this point useful polynomials for polynomial lattice rules can only be found by computer search. In [28] such a search was done based on the quality parameter $t$, that is, the aim was to find polynomials which minimize the $t$-value. Though a small $t$-value yields good distribution properties of the point set, it lacks some flexibility in adjusting the point set to the task at hand. We will see later on that our algorithm does not have this drawback.

Many articles considered the worst-case error of integration in certain function spaces, especially tensor product Hilbert spaces equipped with a reproducing kernel (for more information on reproducing kernels see 1]). In [32, Sloan and Woźniakowski introduced a sequence $\gamma:=\left(\gamma_{j}\right)_{j \geq 1}$ of non-negative reals called weights, with the intention of having spaces where different coordinates can have different importance for the integration error. This flexibility allows us to adjust point sets more accurately to certain problems by choosing appropriate weights (see [6, 7, 36, 37]) and therefore also to improve the performance of the underlying QMC algorithm. Thus also the construction of the underlying point set should be flexible enough to adjust them to the weights. Such construction methods for lattice rules are known, namely the construction by Korobov [10, 11, 38, and by Sloan and his collaborators [4, 12, 30, 31, and it has been shown that they yield good results. (The underlying point set for lattice rules is given by $\{h \boldsymbol{z} / n\}$ for $h=0, \ldots, n-1$, where $n$ is the number of points (usually $n$ is a prime number), the generating vector $\boldsymbol{z}$ is an integer vector and the braces indicate that we take the fractional part of each component. See [29] for more details about lattice rules.) The construction method is based on an explicit formula for the worst-case error and the generating vector is found by minimizing the worst-case error in some sense. Until now no equivalent method was known for digital nets or, more specifically, for polynomial lattice rules.

Here we introduce a construction for polynomial lattice rules which is analogous to the known constructions for lattice rules. This has been made possible by the recent paper [5]. A suitable weighted Hilbert space $H_{\text {wal }, b, s, \alpha, \gamma}$ based on Walsh functions has been introduced in [5], with an explicit formula for the worst-case error. Here we obtain results for the special case when one uses polynomial lattice rules. Furthermore, we show that in this case the formula for the worst-case error can be computed in $O(n s)$ operations, where $n$ is the number of points and $s$ is the dimension. Therefore we are equipped with the basic ingredients previously used 
for the construction of lattice rules for integration in weighted Korobov spaces. In analogy to lattice rules, we establish a component-by-component construction and a Korobov construction of polynomial lattice rules for integration in weighted Hilbert spaces based on Walsh functions. We also prove upper bounds on the worst-case error showing the usefulness of those construction algorithms.

Randomized quasi-Monte Carlo rules, such as shifted lattice rules or digitally shifted digital nets, have been the center of many papers. The aim of this approach is to combine the advantages of deterministic and random point sets. The crux of such methods is to preserve the structure of the original deterministic point set. Cranley and Patterson 3 considered randomly shifted lattice rules, that is, the underlying point set is given by $\{h \boldsymbol{z} / n+\boldsymbol{\Delta}\}$ for $h=0, \ldots, n-1$, where the shift $\boldsymbol{\Delta} \in[0,1)^{s}$ is chosen randomly. It has been shown that randomly shifted lattice rules can also be used successfully for integration in weighted Sobolev spaces. Hickernell 9] introduced a shift invariant kernel associated to the reproducing kernel. This shift invariant kernel is very similar to the reproducing kernel of a weighted Korobov space. This connection has been used to translate the results obtained for integration in weighted Korobov spaces to the integration problem in weighted Sobolev spaces using randomly shifted lattice rules. An analogous theory for randomly digitally shifted digital nets has been developed by Dick and Pillichshammer. In [5] the digital shift invariant kernel associated to the reproducing kernel of the weighted Sobolev space has been calculated. This digital shift invariant kernel is very similar to the reproducing kernel of the weighted Hilbert space based on Walsh functions; hence all the results for this space can be used to obtain results for the integration problem in weighted Sobolev spaces using randomly digitally shifted digital nets (see [5]). We will show here that this also holds when one uses polynomial lattice rules. Hence we obtain construction algorithms for polynomial lattice rules for integration in weighted Sobolev spaces. Furthermore, upper bounds on the mean square worst-case error for randomly digitally shifted polynomial lattice rules constructed by our algorithms can be obtain from the previous work. These upper bounds are comparable to those on the mean square worst-case error for randomly shifted lattice rules for integration in weighted Sobolev spaces. Also, the construction cost for the algorithms presented here is of the same order as for the construction algorithms for randomly shifted lattice rules for integration in weighted Sobolev spaces.

We end this section with a brief outline of the paper. In the next section we introduce $(t, m, s)$-nets, digital $(t, m, s)$-nets and polynomial lattice rules. In Section 3 we introduce Walsh functions and the weighted Hilbert space $H_{\text {wal }, b, s, \alpha, \gamma}$, which is based on Walsh functions. Section 4 deals with multivariate integration in the space $H_{\text {wal }, b, s, \alpha, \gamma}$ using polynomial lattice rules. A component-by-component algorithm and a Korobov type construction of polynomial lattice rules are introduced and analyzed. Integration in weighted Sobolev spaces is considered in Section 5 , We obtain analogous results to those in Section 4 for multivariate integration in weighted Sobolev spaces using randomly digitally shifted polynomial lattice rules. In Section [ 6 we present numerical results comparing the performance of randomly shifted lattice rules and randomly digitally shifted polynomial lattice rules for integration in weighted Sobolev spaces. 


\section{2. $(t, m, s)$-NETS AND POLYNOMIAL LATTICE RULES}

In this section we recall the definition of (digital) $(t, m, s)$-nets in base $b$ and a special construction of such nets due to Niederreiter. A detailed theory of $(t, m, s)$ nets was developed in Niederreiter [19] (see also [21, Chapter 4] for a survey of this theory). The $(t, m, s)$-nets in base $b$ provide sets of $b^{m}$ points in the $s$-dimensional unit cube $[0,1)^{s}$, which are extremely well distributed if the quality parameter $t$ is small.

Definition $2.1((t, m, s)$-nets). Let $b \geq 2, s \geq 1$ and $0 \leq t \leq m$ be integers. Then a point set $P$ consisting of $b^{m}$ points in $[0,1)^{s}$ forms a $(t, m, s)$-net in base $b$ if every subinterval $J=\prod_{j=1}^{s}\left[a_{j} b^{-d_{j}},\left(a_{j}+1\right) b^{-d_{j}}\right) \subseteq[0,1)^{s}$ of volume $b^{t-m}$, with integers $d_{j} \geq 0$ and integers $0 \leq a_{j}<b^{d_{j}}$ for $1 \leq j \leq s$, contains exactly $b^{t}$ points of $P$.

In practice, all concrete constructions of $(t, m, s)$-nets in base $b$ are based on the general construction scheme of digital nets. To avoid too many technical notions, we restrict ourselves in the following to digital nets defined over the finite field $\mathbb{F}_{b}$ and hence $b$ is restricted to prime powers. For a more general definition (over arbitrary finite commutative rings) see for example Niederreiter [21], Larcher [13], or Larcher, Niederreiter and Schmid [15].

Definition 2.2 (Digital $(t, m, s)$-nets). Let $b$ be a prime-power and let $s \geq 1$ and $m \geq 1$ be integers. Let $C_{1}, \ldots, C_{s}$ be $m \times m$ matrices over the finite field $\mathbb{F}_{b}$. We construct $b^{m}$ points in $[0,1)^{s}$ in the following way: for $0 \leq h<b^{m}$ let $h=h_{0}+h_{1} b+\cdots+h_{m-1} b^{m-1}$ be the $b$-adic expansion of $h$. Consider an arbitrary but fixed bijection $\varphi:\{0,1, \ldots, b-1\} \longrightarrow \mathbb{F}_{b}$. Identify $h$ with the vector $\vec{h}=\left(\varphi\left(h_{0}\right), \ldots, \varphi\left(h_{m-1}\right)\right)^{T} \in \mathbb{F}_{b}^{m}$, where $T$ means the transpose of the vector. For $1 \leq j \leq s$ multiply the matrix $C_{j}$ by $\vec{h}$, i.e.,

$$
C_{j} \vec{h}=:\left(y_{j, 1}(h), \ldots, y_{j, m}(h)\right)^{T} \in \mathbb{F}_{b}^{m},
$$

and set

If the point set

$$
x_{h, j}:=\frac{\varphi^{-1}\left(y_{j, 1}(h)\right)}{b}+\cdots+\frac{\varphi^{-1}\left(y_{j, m}(h)\right)}{b^{m}} .
$$

$$
\left\{\boldsymbol{x}_{h}=\left(x_{h, 1}, \ldots, x_{h, s}\right): 0 \leq h<b^{m}\right\}
$$

is a $(t, m, s)$-net in base $b$ for some integer $t$ with $0 \leq t \leq m$, then it is called a digital $(t, m, s)$-net over $\mathbb{F}_{b}$ or, for short, a digital net (over $\mathbb{F}_{b}$ ).

Concerning the determination of the quality parameter $t$ of digital nets, we refer to Niederreiter [21, Theorem 4.28]; see also [26].

In [20] (see also [21, Section 4.4]) Niederreiter introduced a special family of digital $(t, m, s)$-nets over $\mathbb{F}_{b}$. Those nets are obtained from rational functions over finite fields. For a prime-power $b$ let $\mathbb{F}_{b}\left(\left(x^{-1}\right)\right)$ be the field of formal Laurent series over $\mathbb{F}_{b}$. Elements of $\mathbb{F}_{b}\left(\left(x^{-1}\right)\right)$ are formal Laurent series,

$$
L=\sum_{l=w}^{\infty} t_{l} x^{-l}
$$

where $w$ is an arbitrary integer and all $t_{l} \in \mathbb{F}_{b}$. Note that $\mathbb{F}_{b}\left(\left(x^{-1}\right)\right)$ contains the field of rational functions over $\mathbb{F}_{b}$ as a subfield. Furthermore, let $\mathbb{F}_{b}[x]$ be the set of all polynomials over $\mathbb{F}_{b}$. 
Definition 2.3 (Polynomial lattice rules). For a given dimension $s \geq 1$, choose $p \in \mathbb{F}_{b}[x]$ with $\operatorname{deg}(p)=m \geq 1$ and let $q_{1}, \ldots, q_{s} \in \mathbb{F}_{b}[x]$. For $1 \leq j \leq s$, consider the expansions

$$
\frac{q_{j}(x)}{p(x)}=\sum_{l=w_{j}}^{\infty} u_{l}^{(j)} x^{-l} \in \mathbb{F}_{b}\left(\left(x^{-1}\right)\right)
$$

where $w_{j} \leq 1$. Consider the $m \times m$ matrices $C_{1}, \ldots, C_{s}$ over $\mathbb{F}_{b}$ where the elements $c_{i, r}^{(j)}$ of the matrix $C_{j}$ are given by

$$
c_{i, r}^{(j)}=u_{r+i}^{(j)} \in \mathbb{F}_{b},
$$

for $1 \leq j \leq s, 1 \leq i \leq m, 0 \leq r \leq m-1$ and construct a digital $(t, m, s)$-net over $\mathbb{F}_{b}$ with these $s$ matrices. The digital net given by the polynomials $p$ and $\boldsymbol{q}:=\left(q_{1}, \ldots, q_{s}\right) \in \mathbb{F}_{b}^{s}$ is denoted by $S_{p}(\boldsymbol{q})$. A quasi-Monte Carlo rule using the point set $S_{p}(\boldsymbol{q})$ is called a polynomial lattice rule.

For the determination of the quality parameter $t$ of $S_{p}(\boldsymbol{q})$ we refer to [21, Theorem 4.42]. We continue by stating some properties of the construction of the point set $S_{p}(\boldsymbol{q})$.

Remark 2.4. Note that the matrices defined by (2.1) are of the form

$$
C_{j}=C_{q_{j}, p}=\left(\begin{array}{lllc}
u_{1}^{(j)} & u_{2}^{(j)} & \ldots & u_{m}^{(j)} \\
u_{2}^{(j)} & & . \cdot & \vdots \\
\vdots & . \cdot & & \vdots \\
u_{m}^{(j)} & \ldots & \ldots & u_{2 m-1}^{(j)}
\end{array}\right),
$$

i.e., the matrix $C_{j}$ is a so-called Hankel matrix associated with the linear recurring sequence $\left(u_{1}^{(j)}, u_{2}^{(j)}, \ldots\right)$. If $\operatorname{gcd}\left(q_{j}, p\right)=1$, then $p$ is called the minimal polynomial of the linear recurring sequence (see for example [21, Appendix $\mathrm{A}]$ ) and $C_{j}$ is nonsingular (see [17, Theorem 6.75]).

Remark 2.5. From Remark 2.4 it follows that if $\operatorname{gcd}\left(q_{j}, p\right)=1$ for all $1 \leq j \leq s$, then each one-dimensional projection of the point set $S_{p}(\boldsymbol{q})$ to the $j$-th coordinate is a $(0, m, 1)$-net over $\mathbb{F}_{b}$.

The following result concerning the determination of the Laurent series coefficients of rational functions was already stated in [16, 26, 28] for the case $b=2$.

Proposition 2.6. For $p \in \mathbb{F}_{b}[x], p=x^{m}+p_{1} x^{m-1}+\cdots+p_{m-1} x+p_{m}$ and $q \in \mathbb{F}_{b}[x]$ with $q(x)=c_{1} x^{m-1}+\cdots+c_{m-1} x+c_{m}$, the coefficients $u_{l}, l \geq 1$, in the Laurent series expansion of

$$
\frac{q(x)}{p(x)}=\sum_{l=1}^{\infty} u_{l} x^{-l}
$$

can be computed as follows: the first $m$ coefficients $u_{1}, \ldots, u_{m}$ are obtained by solving the linear system

$$
\left(\begin{array}{cccc}
1 & 0 & \ldots & 0 \\
p_{1} & \ddots & \ddots & \vdots \\
\vdots & \ddots & \ddots & 0 \\
p_{m-1} & \ldots & p_{1} & 1
\end{array}\right)\left(\begin{array}{c}
u_{1} \\
u_{2} \\
\vdots \\
u_{m}
\end{array}\right)=\left(\begin{array}{c}
c_{1} \\
c_{2} \\
\vdots \\
c_{m}
\end{array}\right)
$$


and for $l>m, u_{l}$ is obtained from the linear recursion in $\mathbb{F}_{b}$,

$$
u_{l}+u_{l-1} p_{1}+u_{l-2} p_{2}+\cdots+u_{l-m} p_{m}=0 .
$$

Proof. Consider $q(x)=p(x) \sum_{l=1}^{\infty} u_{l} x^{-l}$ and compare the coefficients of $x^{l}, l \in \mathbb{Z}$, on both sides of the equation.

Remark 2.7. If $b$ is a prime, then there is an equivalent but simpler form of the construction of the point set $S_{p}(\boldsymbol{q})$; see [22. For an integer $m \geq 1$ let $v_{m}$ be the map from $\mathbb{F}_{b}\left(\left(x^{-1}\right)\right)$ to the interval $[0,1)$ defined by

$$
v_{m}\left(\sum_{l=w}^{\infty} t_{l} x^{-l}\right)=\sum_{l=\max (1, w)}^{m} t_{l} b^{-l} .
$$

Choose $p$ and $\boldsymbol{q}$ as above. Since $b$ is prime, $\{0, \ldots, b-1\}$ and $\mathbb{F}_{b}$ can be identified and so the bijection $\varphi$ from Definition 2.2 can be taken as the identity map. For $0 \leq h<b^{m}$ let $h=h_{0}+h_{1} b+\cdots+h_{m-1} b^{m-1}$ be the $b$-adic expansion of $h$. With each such $h$ we associate the polynomial

$$
h(x)=\sum_{r=0}^{m-1} h_{r} x^{r} \in \mathbb{F}_{b}[x] .
$$

Then $S_{p}(\boldsymbol{q})$ is the point set consisting of the $b^{m}$ points

$$
\boldsymbol{x}_{h}=\left(v_{m}\left(\frac{h(x) q_{1}(x)}{p(x)}\right), \ldots, v_{m}\left(\frac{h(x) q_{s}(x)}{p(x)}\right)\right) \in[0,1)^{s},
$$

for $0 \leq h<b^{m}$.

Finally we introduce some notation: for arbitrary $\boldsymbol{k}=\left(k_{1}, \ldots, k_{s}\right) \in \mathbb{F}_{b}[x]^{s}$ and $\boldsymbol{q}=\left(q_{1}, \ldots, q_{s}\right) \in \mathbb{F}_{b}[x]^{s}$, we define the 'inner product'

$$
\boldsymbol{k} \cdot \boldsymbol{q}=\sum_{j=1}^{s} k_{j} q_{j} \in \mathbb{F}_{b}[x]
$$

and we write $q \equiv 0(\bmod p)$ if $p$ divides $q$ in $\mathbb{F}_{b}[x]$. Furthermore, as in Remark 2.7 for $b$ prime we associate a non-negative integer $k=\kappa_{0}+\kappa_{1} b+\cdots+\kappa_{a} b^{a}$ with the polynomial $k(x)=\kappa_{0}+\kappa_{1} x+\cdots+\kappa_{a} x^{a} \in \mathbb{F}_{b}[x]$.

\section{Weighted Hilbert spaces Based on Walsh functions}

In the following subsection we recall the definition of Walsh functions and in Subsection 3.2 we introduce the weighted Hilbert space based on Walsh functions first defined in [5]. Henceforth let $\mathbb{N}_{0}$ denote the set of non-negative integers.

3.1. Walsh functions. We have the following definitions.

Definition 3.1 (Walsh functions). Let $b \geq 2$ be an integer. For a non-negative integer $k$ with base $b$ representation

$$
k=\kappa_{0}+\kappa_{1} b+\cdots+\kappa_{a} b^{a},
$$

with $\kappa_{i} \in\{0, \ldots, b-1\}$, we define the Walsh function ${ }_{b}$ wal $_{k}:[0,1) \longrightarrow \mathbb{C}$ by

$$
{ }_{b} \operatorname{wal}_{k}(x):=e^{2 \pi \mathrm{i}\left(x_{1} \kappa_{0}+\cdots+x_{a+1} \kappa_{a}\right) / b},
$$

for $x \in[0,1)$ with base $b$ representation $x=\frac{x_{1}}{b}+\frac{x_{2}}{b^{2}}+\cdots$ (unique in the sense that infinitely many of the $x_{i}$ must be different from $b-1$ ). If it is clear which base $b$ is chosen, we will simply write wal $_{k}$. 
Definition 3.2 (Multivariate Walsh functions). For dimension $s \geq 2, x_{1}, \ldots, x_{s} \in$ $[0,1)$ and $k_{1}, \ldots, k_{s} \in \mathbb{N}_{0}$ we define ${ }_{b}$ wal $_{k_{1}, \ldots, k_{s}}:[0,1)^{s} \longrightarrow \mathbb{C}$ by

$$
{ }_{b} \operatorname{wal}_{k_{1}, \ldots, k_{s}}\left(x_{1}, \ldots, x_{s}\right):=\prod_{j=1}^{s} b \operatorname{wal}_{k_{j}}\left(x_{j}\right) .
$$

For vectors $\boldsymbol{k}=\left(k_{1}, \ldots, k_{s}\right) \in \mathbb{N}_{0}^{s}$ and $\boldsymbol{x}=\left(x_{1}, \ldots, x_{s}\right) \in[0,1)^{s}$ we write

$$
{ }_{b} \operatorname{wal}_{\boldsymbol{k}}(\boldsymbol{x}):={ }_{b} \mathrm{wal}_{k_{1}, \ldots, k_{s}}\left(x_{1}, \ldots, x_{s}\right) .
$$

Again, if it is clear which base we mean, we simply write wal $_{\boldsymbol{k}}(\boldsymbol{x})$.

It is clear from the definitions that Walsh functions are piecewise constant. It can be shown that for any integers $s \geq 1$ and $b \geq 2$ the system $\left\{b\right.$ wal $_{k_{1}, \ldots, k_{s}}$ : $\left.k_{1}, \ldots, k_{s} \geq 0\right\}$ is a complete orthonormal system in $L_{2}\left([0,1)^{s}\right)$; see for example [2, 18] or [25, Satz 1]. More information on Walsh functions can be found for example in 2, 25, 27, 35.

3.2. The Hilbert space $H_{\mathrm{wal}, b, s, \alpha, \boldsymbol{\gamma}}$. In the following we define the weighted Hilbert space $H_{\mathrm{wal}, b, s, \alpha, \gamma}$ which is based on Walsh functions (see [5]). First we consider the one-dimensional case. The $s$-dimensional space will then be defined as the tensor product of those one-dimensional spaces.

For a natural number $k=\kappa_{0}+\kappa_{1} b+\cdots+\kappa_{a} b^{a}$, with $\kappa_{a} \neq 0$, let $\psi_{b}(k)=a$. For $\alpha>1$ we define

$$
r_{b}(\alpha, \gamma, k)= \begin{cases}1, & \text { if } k=0, \\ \gamma b^{-\alpha \psi_{b}(k)}, & \text { if } k \neq 0 .\end{cases}
$$

Furthermore, we define the inner product of two functions $f$ and $g$ as

$$
\langle f, g\rangle_{\mathrm{wal}, b, \alpha, \gamma}:=\sum_{k \in \mathbb{N}_{0}} r_{b}(\alpha, \gamma, k)^{-1} \hat{f}_{\mathrm{wal}}(k) \overline{\hat{g}_{\mathrm{wal}}(k)},
$$

where

$$
\hat{f}_{\mathrm{wal}}(k)=\int_{0}^{1} f(x) \overline{b \mathrm{wal}_{k}(x)} \mathrm{d} x .
$$

The norm is given by $\|f\|_{\text {wal }, b, \alpha, \gamma}:=\langle f, f\rangle_{\text {wal }, b, \alpha, \gamma}^{1 / 2}$. Note that any function $f \in$ $L_{2}([0,1))$ can be written as

$$
f(x)=\sum_{k=0}^{\infty} \hat{f}_{\mathrm{wal}}(k) \mathrm{wal}_{k}(x) .
$$

The weighted Hilbert space $H_{\text {wal }, b, \alpha, \gamma}$ is now given by all functions with finite norm, that is,

$$
H_{\mathrm{wal}, b, \alpha, \gamma}:=\left\{f:\|f\|_{\mathrm{wal}, b, \alpha, \gamma}<\infty\right\} .
$$

It can be checked (see [5, Subsection 2.2]) that $H_{\text {wal }, b, \alpha, \gamma}$ is a reproducing kernel Hilbert space with reproducing kernel given by

$$
K_{\text {wal }, b, \alpha, \gamma}(x, y)=\sum_{k \in \mathbb{N}_{0}} r_{b}(\alpha, \gamma, k)_{b} \operatorname{wal}_{k}(x) \overline{b \mathrm{wal}_{k}(y)} .
$$

This reproducing kernel can be simplified in the following way: for $\alpha>1$ we define

$$
\mu_{b}(\alpha):=\sum_{k=1}^{\infty} b^{-\alpha \psi_{b}(k)}
$$


and a simple calculation yields

$$
\mu_{b}(\alpha)=\sum_{a=0}^{\infty} b^{-\alpha a}(b-1) b^{a}=\frac{b^{\alpha}(b-1)}{b^{\alpha}-b} .
$$

For $x=\frac{x_{1}}{b}+\frac{x_{2}}{b^{2}}+\cdots$ and $y=\frac{y_{1}}{b}+\frac{y_{2}}{b^{2}}+\cdots$ we define

$$
\begin{aligned}
& \phi_{\mathrm{wal}, b, \alpha}(x, y) \\
& \quad= \begin{cases}\mu_{b}(\alpha), & \text { if } x=y, \\
\mu_{b}(\alpha)-b^{\left(i_{0}-1\right)(1-\alpha)}\left(\mu_{b}(\alpha)+1\right), & \text { if } x_{i_{0}} \neq y_{i_{0}} \text { and } x_{i}=y_{i}\end{cases} \\
& \text { for } i=1, \ldots, i_{0}-1,
\end{aligned}
$$

with $\mu_{b}$ given by (3.2). Note that the function values of $\phi_{\mathrm{wal}, b, \alpha}$ can easily be computed for any $x$ and $y$ and all $\alpha>1$. Then in [5] it was proved that

$$
K_{\mathrm{wal}, b, \alpha, \gamma}(x, y)=1+\gamma \phi_{\mathrm{wal}, b, \alpha}(x, y) .
$$

We are now ready to consider the $s$-dimensional case. Let $\gamma=\left(\gamma_{j}\right)_{j \geq 1}$ be a sequence of non-negative real numbers. The weighted Hilbert space $H_{\mathrm{wal}, b, s, \alpha, \gamma}$ in base $b$ and dimension $s$ is now the tensor product of the one-dimensional weighted Hilbert spaces $H_{\mathrm{wal}, b, \alpha, \gamma_{j}}$, that is,

$$
H_{\mathrm{wal}, b, s, \alpha, \gamma}=H_{\mathrm{wal}, b, \alpha, \gamma_{1}} \otimes \cdots \otimes H_{\mathrm{wal}, b, \alpha, \gamma_{s}} .
$$

Let $\boldsymbol{k}=\left(k_{1}, \ldots, k_{s}\right)$ and $\boldsymbol{x}$ and $\boldsymbol{y}$ be defined analogously. The space $H_{\mathrm{wal}, b, s, \alpha, \boldsymbol{\gamma}}$ is again a reproducing kernel Hilbert space, with reproducing kernel given by

$$
\begin{aligned}
K_{\mathrm{wal}, b, s, \alpha, \boldsymbol{\gamma}}(\boldsymbol{x}, \boldsymbol{y}) & =\prod_{j=1}^{s} K_{\mathrm{wal}, b, \alpha, \gamma_{j}}\left(x_{j}, y_{j}\right) \\
& =\prod_{j=1}^{s} \sum_{k_{j}=0}^{\infty} r_{b}\left(\alpha, \gamma_{j}, k_{j}\right)_{b} \mathrm{wal}_{k_{j}}\left(x_{j}\right) \overline{b \mathrm{wal}_{k_{j}}\left(y_{j}\right)} \\
& =\sum_{\boldsymbol{k} \in \mathbb{N}_{0}^{s}} r_{b}(\alpha, \gamma, \boldsymbol{k})_{b} \mathrm{wal}_{\boldsymbol{k}}(\boldsymbol{x}) \overline{{ }_{b} \mathrm{wal}_{\boldsymbol{k}}(\boldsymbol{y})}
\end{aligned}
$$

where $r_{b}(\alpha, \gamma, \boldsymbol{k})=\prod_{j=1}^{s} r_{b}\left(\alpha, \gamma_{j}, k_{j}\right)$. The reproducing kernel can also be written as

$$
K_{\mathrm{wal}, b, s, \alpha, \boldsymbol{\gamma}}(\boldsymbol{x}, \boldsymbol{y})=\prod_{j=1}^{s}\left(1+\gamma_{j} \phi_{\mathrm{wal}, b, \alpha}\left(x_{j}, y_{j}\right)\right),
$$

where $\phi_{\mathrm{wal}, b, \alpha}$ is given by (3.3). (Hence $K_{\mathrm{wal}, b, s, \alpha, \gamma}$ can be computed easily.) The inner product in $H_{\mathrm{wal}, b, s, \alpha, \gamma}$ is given by

$$
\langle f, g\rangle_{\mathrm{wal}, b, s, \alpha, \boldsymbol{\gamma}}=\sum_{\boldsymbol{k} \in \mathbb{N}_{0}^{s}} r_{b}(\alpha, \boldsymbol{\gamma}, \boldsymbol{k})^{-1} \hat{f}_{\mathrm{wal}}(\boldsymbol{k}) \overline{\hat{g}_{\mathrm{wal}}(\boldsymbol{k})},
$$

where

$$
\hat{f}_{\text {wal }}(\boldsymbol{k}):=\int_{[0,1)^{s}} f(\boldsymbol{x}) \overline{b \mathrm{wal}_{\boldsymbol{k}}(\boldsymbol{x})} \mathrm{d} x .
$$

In the following, if not necessary, the dependence of the weighted Hilbert space $H_{\text {wal }, b, s, \alpha, \gamma}$ on some of the parameters $b, s, \alpha, \gamma$ is not expressed explicitly. The same also holds accordingly for $r,\langle\cdot, \cdot\rangle,\|\cdot\|, K, \mu$ and $\phi$. 


\section{Multivariate integration in Weighted Hilbert spaces BASED ON WALSH FUNCTIONS}

In this section we introduce and analyze two construction algorithms for polynomial lattice rules based on the worst-case error; see Subsections 4.2 and 4.3. A formula for the worst-case error is presented in the following subsection.

Henceforth, in order to have simple notation, we restrict $b$ to prime numbers. In this case the finite field $\mathbb{F}_{b}$ is just $\mathbb{Z}_{b}$, the least residue ring modulo $b$, and the bijections in Definition 2.2 can be chosen as identities. We remark that the subsequent results can also be obtained for arbitrary finite fields $\mathbb{F}_{b}$.

4.1. Multivariate integration in $H_{\mathrm{wal}, b, s, \alpha, \boldsymbol{\gamma}}$. In general we are interested in approximating the integrals of functions $f$ from a reproducing kernel Hilbert space $H$,

$$
I_{s}(f)=\int_{[0,1)^{s}} f(\boldsymbol{x}) \mathrm{d} \boldsymbol{x} .
$$

We approximate the integral $I_{s}(f)$ by quasi-Monte Carlo (QMC) algorithms, which are equal weight quadrature rules of the form

$$
Q_{n, s}\left(P_{n}, f\right)=\frac{1}{n} \sum_{h=0}^{n-1} f\left(\boldsymbol{x}_{h}\right)
$$

with a deterministically chosen point set $P_{n}=\left\{\boldsymbol{x}_{0}, \ldots, \boldsymbol{x}_{n-1}\right\}$.

The worst-case error of a QMC rule $Q_{n, s}$ for integration in the space $H$ with reproducing kernel $K$ and norm $\|\cdot\|$ is defined by

$$
e\left(P_{n}, K\right):=\sup _{f \in H,\|f\| \leq 1}\left|I_{s}(f)-Q_{n, s}\left(P_{n}, f\right)\right|,
$$

and the initial error is

$$
e_{0, s}:=\sup _{f \in H,\|f\| \leq 1}\left|I_{s}(f)\right| .
$$

For the space $H_{\text {wal }, b, s, \alpha, \boldsymbol{\gamma}}$ we have $I_{s}(f)=\hat{f}_{\text {wal }}(\mathbf{0})=\langle f, 1\rangle_{\text {wal }, b, s, \alpha, \boldsymbol{\gamma}}$. Thus the representer of the functional $I_{s}(f)$ in the reproducing kernel Hilbert space $H_{\text {wal }, b, s, \alpha, \gamma}$ is the function 1 , and it follows immediately that the initial error is given by

$$
e_{0, s}=\|1\|_{\mathrm{wal}, b, s, \alpha, \boldsymbol{\gamma}}=1 .
$$

Let $C_{1}, \ldots, C_{s}$ denote the generating matrices for a digital $(t, m, s)$-net over $\mathbb{Z}_{b}$. It was shown in [5] that the worst-case error in $H_{\mathrm{wal}, b, s, \alpha, \gamma}$ using such a digital net satisfies

$$
e_{b^{m}, s}^{2}\left(C_{1}, \ldots, C_{s}\right)=\sum_{\boldsymbol{k} \in D} r_{b}(\alpha, \gamma, \boldsymbol{k})
$$

where

$$
D=\left\{\boldsymbol{k} \in \mathbb{N}_{0}^{s} \backslash\{\mathbf{0}\}: C_{1}^{T} \operatorname{tr}_{m}\left(\vec{k}_{1}\right)+\cdots+C_{s}^{T} \operatorname{tr}_{m}\left(\vec{k}_{s}\right)=\overrightarrow{0}\right\}
$$

Here for a non-negative integer $k$ with $b$-adic expansion $k=\kappa_{0}+\kappa_{1} b+\cdots$, we write

$$
\vec{k}=\left(\kappa_{0}, \kappa_{1}, \ldots\right)^{T},
$$

which is an infinite-dimensional vector, and we use

$$
\operatorname{tr}_{m}(\vec{k})=\left(\kappa_{0}, \kappa_{1}, \ldots, \kappa_{m-1}\right)^{T}
$$


to denote the $m$-dimensional vector containing the first $m$ components of $\vec{k}$. Furthermore, it was proved in [5] that

$$
e_{b^{m}, s}^{2}\left(C_{1}, \ldots, C_{s}\right)=-1+\frac{1}{b^{m}} \sum_{h=0}^{b^{m}-1} \prod_{j=1}^{s}\left(1+\gamma_{j} \phi_{\mathrm{wal}, \alpha}\left(x_{h, j}, 0\right)\right),
$$

where $\phi_{\mathrm{wal}, \alpha}$ is given by (3.3), and $\boldsymbol{x}_{0}, \ldots, \boldsymbol{x}_{b^{m}-1}$ are the points of the digital net, with $\boldsymbol{x}_{h}=\left(x_{h, 1}, \ldots, x_{h, s}\right)$.

Expression (4.2) allows us to compute $e_{b^{m}, s}^{2}\left(C_{1}, \ldots, C_{s}\right)$ for any given digital net in $O\left(b^{m} s\right)$ operations. We exploit this fact by using a computer search to find digital nets which yield a small worst-case error. A similar approach was used for lattice rules; see for example [10, 12, 30, 31]. We remark that in our construction of digital $(t, m, s)$-nets we seek to minimize (in some sense) the worst-case error. Note that this approach is different from the classical approach where one tries to find digital nets with minimal $t$-value.

4.2. The component-by-component construction of polynomial lattice rules for integration in $H_{\mathrm{wal}, b, s, \alpha, \gamma}$. In this subsection we introduce a construction algorithm for polynomial lattice rules.

For a non-negative integer $k$ with $b$-adic expansion $k=\kappa_{0}+\kappa_{1} b+\cdots$, we write

$$
\operatorname{tr}_{m}(k)=\kappa_{0}+\kappa_{1} b+\cdots+\kappa_{m-1} b^{m-1} ;
$$

thus the associated polynomial $\operatorname{tr}_{m}(k)(x)=\kappa_{0}+\kappa_{1} x+\cdots+\kappa_{m-1} x^{m-1} \in \mathbb{Z}_{b}[x]$ has degree $<m$. For a vector $\boldsymbol{k} \in \mathbb{N}_{0}^{s}, \operatorname{tr}_{m}(\boldsymbol{k})$ is defined componentwise. In the following lemma $\operatorname{tr}_{m}(\boldsymbol{k})$ is considered as a vector of polynomials in $\mathbb{Z}_{b}[x]$; see Section 2

Lemma 4.1. Let $p \in \mathbb{Z}_{b}[x]$ with $\operatorname{deg}(p)=m \geq 1$ and let $\boldsymbol{q}=\left(q_{1}, \ldots, q_{s}\right) \in \mathbb{Z}_{b}[x]^{s}$. Let $\left\{\boldsymbol{x}_{0}, \ldots, \boldsymbol{x}_{b^{m}-1}\right\}$ be the point set $S_{p}(\boldsymbol{q})$. Then the square worst-case error for integration in the weighted Hilbert space $H_{\mathrm{wal}, b, s, \alpha, \gamma}$ is given by

$$
e_{b^{m}, s}^{2}(\boldsymbol{q}, p)=\sum_{\boldsymbol{k} \in D} r_{b}(\alpha, \boldsymbol{\gamma}, \boldsymbol{k})
$$

where

$$
D=\left\{\boldsymbol{k} \in \mathbb{N}_{0}^{s} \backslash\{\mathbf{0}\}: \operatorname{tr}_{m}(\boldsymbol{k}) \cdot \boldsymbol{q} \equiv 0(\bmod p)\right\} .
$$

Remark 4.2. Compare the above lemma with [33, formula (15)], which states that the squared worst-case error $\bar{e}_{n, s}^{2}$ for integration in the weighted Korobov space using an $n$ point lattice rule with generating vector $\boldsymbol{z}$ is given by

$$
\bar{e}_{n, s}^{2}(\boldsymbol{z})=\sum_{\boldsymbol{k} \in \mathcal{L}} \bar{r}(\alpha, \boldsymbol{\gamma}, \boldsymbol{k}),
$$

where $\mathcal{L}=\left\{\boldsymbol{k} \in \mathbb{Z}^{s} \backslash\{\mathbf{0}\}: \boldsymbol{k} \cdot \boldsymbol{z} \equiv 0(\bmod n)\right\}$ and $\bar{r}(\alpha, \boldsymbol{\gamma}, \boldsymbol{k})=\prod_{j=1}^{s} \bar{r}\left(\alpha, \gamma_{j}, k_{j}\right)$ with $\bar{r}(\alpha, \gamma, 0)=1$ and $\bar{r}(\alpha, \gamma, k)=\gamma|k|^{-\alpha}$ for $k \neq 0$. The set $\mathcal{L}$ is called the dual lattice (see [29]); accordingly we call $D$ the dual polynomial lattice.

Proof. The result follows from the fact that if $C_{1}, \ldots, C_{s}$ are generating matrices for the point set $S_{p}(\boldsymbol{q})$, then for any $\boldsymbol{k}=\left(k_{1}, \ldots, k_{s}\right) \in \mathbb{N}_{0}^{s} \backslash\{\mathbf{0}\}$ we have

$$
C_{1}^{T} \operatorname{tr}_{m}\left(\vec{k}_{1}\right)+\cdots+C_{s}^{T} \operatorname{tr}_{m}\left(\vec{k}_{s}\right)=\overrightarrow{0} \quad \text { iff } \quad \operatorname{tr}_{m}(\boldsymbol{k}) \cdot \boldsymbol{q} \equiv 0(\bmod p) .
$$

This was first proved in [21, Lemma 4.40]. 
Let $R_{b, m}$ be the set of all non-zero polynomials in $\mathbb{Z}_{b}[x]$ with degree at most $m-1$, that is,

$$
R_{b, m}:=\left\{q \in \mathbb{Z}_{b}[x]: \operatorname{deg}(q)<m \text { and } q \neq 0\right\} .
$$

Obviously we have $\left|R_{b, m}\right|=b^{m}-1$.

It follows from the construction principle presented in Section 2 that the polynomials $q_{j}$ can be restricted to the set $R_{b, m}$. Using (4.2), we can therefore use computers to search for good polynomials $q_{j}$. In the following we present an efficient algorithm for such a computer search.

Algorithm 4.3. Given a prime number $b$, a dimension $s$, an integer $m \geq 1$ and weights $\gamma=\left(\gamma_{j}\right)_{j \geq 1}$ :

(1) Choose an irreducible polynomial $p \in \mathbb{Z}_{b}[x]$ with $\operatorname{deg}(p)=m$.

(2) Set $q_{1}=1$.

(3) For $d=2,3, \ldots, s$, find $q_{d} \in R_{b, m}$ by minimizing the square worst-case error $e_{b^{m}, d}^{2}\left(\left(q_{1}, \ldots, q_{d}\right), p\right)$.

In the following we will make use of Jensen's inequality, which states that for a sequence $\left(a_{k}\right)$ of non-negative real numbers we have

$$
\left(\sum a_{k}\right)^{\lambda} \leq \sum a_{k}^{\lambda}
$$

for any $0<\lambda \leq 1$.

Theorem 4.4. Let $b$ be prime and $p \in \mathbb{Z}_{b}[x]$ be irreducible, with $\operatorname{deg}(p)=m \geq 1$. Suppose $\left(q_{1}^{*}, \ldots, q_{s}^{*}\right) \in R_{b, m}^{s}$ is constructed by Algorithm 4.3. Then for all $d=$ $1,2, \ldots, s$ we have

$$
e_{b^{m}, d}^{2}\left(\left(q_{1}^{*}, \ldots, q_{d}^{*}\right), p\right) \leq\left(b^{m}-1\right)^{-\frac{1}{\lambda}} \prod_{j=1}^{d}\left(1+\mu(\alpha \lambda) \gamma_{j}^{\lambda}\right)^{\frac{1}{\lambda}},
$$

for all $\frac{1}{\alpha}<\lambda \leq 1$.

Proof. It is not hard to show that the result is true for $d=1$. Suppose for some $1 \leq d<s$ we have $\boldsymbol{q}^{*} \in R_{b, m}^{d}$ and

$$
e_{b^{m}, d}^{2}\left(\boldsymbol{q}^{*}, p\right) \leq\left(b^{m}-1\right)^{-\frac{1}{\lambda}} \prod_{j=1}^{d}\left(1+\mu(\alpha \lambda) \gamma_{j}^{\lambda}\right)^{\frac{1}{\lambda}},
$$

for all $\frac{1}{\alpha}<\lambda \leq 1$. Now we consider $e_{b^{m}, d+1}^{2}\left(\left(\boldsymbol{q}^{*}, q_{d+1}\right), p\right)$. It follows from Lemma 4.1 that

$$
\begin{array}{ll}
e_{b^{m}, d+1}^{2}\left(\left(\boldsymbol{q}^{*}, q_{d+1}\right), p\right)= & \sum_{\substack{\left(\boldsymbol{k}, k_{d+1}\right) \in \mathbb{N}_{0}^{d+1} \backslash\{\mathbf{0}\} \\
\operatorname{tr}_{m}\left(\boldsymbol{k}, k_{d+1}\right) \cdot\left(\boldsymbol{q}^{*}, q_{d+1}\right) \equiv 0(\bmod p)}} r_{b}(\alpha, \boldsymbol{\gamma}, \boldsymbol{k}) r_{b}\left(\alpha, \gamma_{d+1}, k_{d+1}\right) \\
= & e_{b^{m}, d}^{2}\left(\boldsymbol{q}^{*}, p\right)+\theta\left(q_{d+1}\right),
\end{array}
$$

where we have separated out the $k_{d+1}=0$ terms, and

$$
\theta\left(q_{d+1}\right)=\sum_{k_{d+1}=1}^{\infty}\left(r_{b}\left(\alpha, \gamma_{d+1}, k_{d+1}\right) \sum_{\substack{\boldsymbol{k} \in \mathbb{N}_{0}^{d} \\ \operatorname{tr}_{m}(\boldsymbol{k}) \cdot \boldsymbol{q} \equiv-\operatorname{tr}_{m}\left(k_{d+1}\right) \cdot q_{d+1}(\bmod p)}} r_{b}(\alpha, \boldsymbol{\gamma}, \boldsymbol{k})\right) .
$$


We see from the algorithm that $q_{d+1}^{*}$ is chosen such that the square worst-case error $e_{b^{m}, d+1}^{2}\left(\left(\boldsymbol{q}^{*}, q_{d+1}\right), p\right)$ is minimized. Since the only dependency on $q_{d+1}$ is in $\theta\left(q_{d+1}\right)$, we have $\theta\left(q_{d+1}^{*}\right) \leq \theta\left(q_{d+1}\right)$ for all $q_{d+1} \in R_{b, m}$, which implies that for any $\lambda \leq 1$ we have

$$
\left[\theta\left(q_{d+1}^{*}\right)\right]^{\lambda} \leq\left[\theta\left(q_{d+1}\right)\right]^{\lambda} \quad \text { for all } q_{d+1} \in R_{b, m}
$$

This leads to

$$
\theta\left(q_{d+1}^{*}\right) \leq\left(\frac{1}{b^{m}-1} \sum_{q_{d+1} \in R_{b, m}}\left[\theta\left(q_{d+1}\right)\right]^{\lambda}\right)^{\frac{1}{\lambda}}
$$

We will obtain a bound on $\theta\left(q_{d+1}^{*}\right)$ through this last inequality.

Let $\lambda$ satisfy $\frac{1}{\alpha}<\lambda \leq 1$. It follows from Jensen's inequality and the property $\left[r_{b}(\alpha, \gamma, k)\right]^{\lambda}=r_{b}\left(\alpha \lambda, \gamma^{\lambda}, k\right)$ that

$$
\left[\theta\left(q_{d+1}\right)\right]^{\lambda} \leq \sum_{k_{d+1}=1}^{\infty}\left(r_{b}\left(\alpha \lambda, \gamma_{d+1}^{\lambda}, k_{d+1}\right) \sum_{\substack{k \in \mathbb{N}_{0}^{d} \\ \operatorname{tr}_{m}(\boldsymbol{k}) \cdot \boldsymbol{q} \equiv-\operatorname{tr}_{m}\left(k_{d+1}\right) \cdot q_{d+1}(\bmod p)}} r_{b}\left(\alpha \lambda, \gamma^{\lambda}, \boldsymbol{k}\right)\right)
$$

where $\gamma^{\lambda}$ denotes the sequence $\left(\gamma_{j}^{\lambda}\right)_{j \geq 1}$. If $k_{d+1}$ is a multiple of $b^{m}, \operatorname{then}^{\operatorname{tr}_{m}}\left(k_{d+1}\right)$ $=0$ and the corresponding term in the sum is independent of $q_{d+1}$. If $k_{d+1}$ is not a multiple of $b^{m}$, then $\operatorname{tr}_{m}\left(k_{d+1}\right)$ can have any value between 1 and $b^{m}-1$. Moreover, since $q_{d+1} \neq 0$ and $p$ is irreducible, $\operatorname{tr}_{m}\left(k_{d+1}\right) \cdot q_{d+1}$ is never a multiple of $p$.

By averaging over all $q_{d+1} \in R_{b, m}$, with the above discussion in mind, we obtain

$$
\begin{aligned}
& \frac{1}{b^{m}-1} \sum_{\substack{q_{d+1} \in R_{b, m} \\
\leq}}\left[\theta\left(q_{d+1}\right)\right]^{\lambda} \\
& \sum_{\substack{k_{d+1}=1 \\
b^{m} \mid k_{d+1}}}^{\infty} r_{b}\left(\alpha \lambda, \gamma_{d+1}^{\lambda}, k_{d+1}\right) \sum_{\substack{\boldsymbol{k} \in \mathbb{N}_{0}^{d} \\
\operatorname{tr}_{m}(\boldsymbol{k}) \cdot \boldsymbol{q} \equiv 0(\bmod p)}} r_{b}\left(\alpha \lambda, \gamma^{\lambda}, \boldsymbol{k}\right) \\
& \quad+\frac{1}{b^{m}-1} \sum_{\substack{k_{d+1}=1 \\
b^{m} \nmid k_{d+1}}}^{\infty} r_{b}\left(\alpha \lambda, \gamma_{d+1}^{\lambda}, k_{d+1}\right) \sum_{\substack{\boldsymbol{k} \in \mathbb{N}_{0}^{d} \\
\operatorname{tr}_{m}(\boldsymbol{k}) \cdot \boldsymbol{q} \neq 0(\bmod p)}} r_{b}\left(\alpha \lambda, \gamma^{\lambda}, \boldsymbol{k}\right) \\
& \leq \frac{\mu(\alpha \lambda) \gamma_{d+1}^{\lambda}}{b^{m}-1} \prod_{j=1}^{d}\left(1+\mu(\alpha \lambda) \gamma_{j}^{\lambda}\right),
\end{aligned}
$$

where the first inequality follows from the fact that if $k_{d+1}$ is not a multiple of $b^{m}$, then

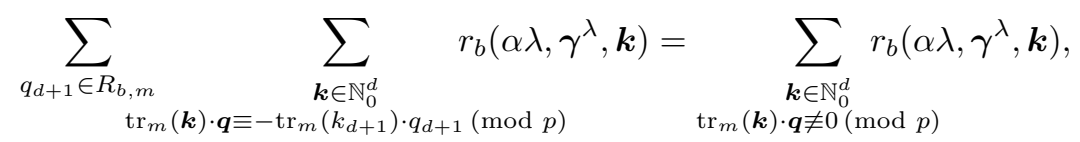


and the second inequality is obtained using the following:

$$
\begin{aligned}
\sum_{\substack{k_{d+1}=1 \\
b^{m} \mid k_{d+1}}}^{\infty} r_{b}\left(\alpha \lambda, \gamma_{d+1}^{\lambda}, k_{d+1}\right) & =\frac{\mu(\alpha \lambda) \gamma_{d+1}^{\lambda}}{b^{\alpha \lambda m}}, \\
\sum_{\substack{k_{d+1}=1 \\
b^{m} \nmid k_{d+1}}}^{\infty} r_{b}\left(\alpha \lambda, \gamma_{d+1}^{\lambda}, k_{d+1}\right) & \leq \sum_{k_{d+1}=1}^{\infty} r_{b}\left(\alpha \lambda, \gamma_{d+1}^{\lambda}, k_{d+1}\right)=\mu(\alpha \lambda) \gamma_{d+1}^{\lambda}, \\
\sum_{\substack{\boldsymbol{k} \in \mathbb{N}_{0}^{d} \\
\operatorname{tr}_{m}(\boldsymbol{k}) \cdot \boldsymbol{q} \neq 0(\bmod p)}} r_{b}\left(\alpha \lambda, \gamma^{\lambda}, \boldsymbol{k}\right) & =\prod_{j=1}^{d}\left(1+\mu(\alpha \lambda) \gamma_{j}^{\lambda}\right)-\sum_{\substack{\boldsymbol{k} \in \mathbb{N}_{0}^{d} \\
\operatorname{tr}_{m}(\boldsymbol{k}) \cdot \boldsymbol{q} \equiv 0(\bmod p)}} r_{b}\left(\alpha \lambda, \gamma^{\lambda}, \boldsymbol{k}\right) .
\end{aligned}
$$

Thus we have from (4.6) and (4.7) that

$$
\theta\left(q_{d+1}^{*}\right) \leq \mu(\alpha \lambda)^{\frac{1}{\lambda}} \gamma_{d+1}\left(b^{m}-1\right)^{-\frac{1}{\lambda}} \prod_{j=1}^{d}\left(1+\mu(\alpha \lambda) \gamma_{j}^{\lambda}\right)^{\frac{1}{\lambda}},
$$

which, together with (4.4) and (4.5), yields

$$
\begin{aligned}
e_{b^{m}, d+1}^{2} & \left(\left(\boldsymbol{q}^{*}, q_{d+1}^{*}\right), p\right) \\
& =e_{b^{m}, d}^{2}\left(\boldsymbol{q}^{*}, p\right)+\theta\left(q_{d+1}^{*}\right) \\
& \leq\left(1+\mu(\alpha \lambda)^{\frac{1}{\lambda}} \gamma_{d+1}\right)\left(b^{m}-1\right)^{-\frac{1}{\lambda}} \prod_{j=1}^{d}\left(1+\mu(\alpha \lambda) \gamma_{j}^{\lambda}\right)^{\frac{1}{\lambda}} \\
& \leq\left(b^{m}-1\right)^{-\frac{1}{\lambda}} \prod_{j=1}^{d+1}\left(1+\mu(\alpha \lambda) \gamma_{j}^{\lambda}\right)^{\frac{1}{\lambda}} .
\end{aligned}
$$

Hence by induction the result follows for all $d=1,2, \ldots, s$.

Corollary 4.5. Let $b$ be prime, $p \in \mathbb{Z}_{b}[x]$ be irreducible with $\operatorname{deg}(p)=m \geq 1$ and $n=b^{m}$. Suppose $\boldsymbol{q}^{*} \in R_{b, m}^{s}$ is constructed by Algorithm 4.3 .

(1) We have

$$
e_{n, s}\left(\boldsymbol{q}^{*}, p\right) \leq c_{s, \alpha, \boldsymbol{\gamma}, \delta} n^{-\frac{\alpha}{2}+\delta} \quad \text { for all } 0<\delta \leq \frac{\alpha-1}{2},
$$

where

$$
c_{s, \alpha, \boldsymbol{\gamma}, \delta}:=2^{\frac{\alpha}{2}-\delta} \prod_{j=1}^{s}\left(1+\mu\left(\frac{\alpha}{\alpha-2 \delta}\right) \gamma_{j}^{\frac{1}{\alpha-2 \delta}}\right)^{\frac{\alpha}{2}-\delta} .
$$

(2) Suppose

$$
\sum_{j=1}^{\infty} \gamma_{j}^{\frac{1}{\alpha-2 \delta}}<\infty
$$

Then $c_{s, \alpha, \boldsymbol{\gamma}, \delta} \leq c_{\infty, \alpha, \boldsymbol{\gamma}, \delta}<\infty$ and we have

$$
e_{n, s}\left(\boldsymbol{q}^{*}, p\right) \leq c_{\infty, \alpha, \boldsymbol{\gamma}, \delta} n^{-\frac{\alpha}{2}+\delta} \quad \text { for all } 0<\delta \leq \frac{\alpha-1}{2} .
$$

Thus the worst-case error is bounded independently of the dimension. 
(3) Under the assumption

$$
A:=\limsup _{s \rightarrow \infty} \frac{\sum_{j=1}^{s} \gamma_{j}}{\log s}<\infty
$$

we obtain $c_{s, \alpha, \gamma,(\alpha-1) / 2} \leq \widetilde{c}_{\eta} s^{\mu(\alpha)(A+\eta)}$ and therefore

$$
e_{n, s}\left(\boldsymbol{q}^{*}, p\right) \leq \widetilde{c}_{\eta} s^{\mu(\alpha)(A+\eta) / 2} n^{-\frac{1}{2}} \quad \text { for all } \eta>0,
$$

where the constant $\widetilde{c}_{\eta}$ depends only on $\eta$. Thus the worst-case error satisfies a bound which depends only polynomially on the dimension.

Proof. The first part of the theorem follows from Theorem 4.4 by setting $\frac{1}{\lambda}=\alpha-2 \delta$ and noting that $b^{m}-1=n-1 \geq \frac{n}{2}$ for all $n \geq 1$.

Furthermore, we have

$$
\begin{aligned}
\prod_{j=1}^{s}\left(1+\mu\left(\frac{\alpha}{\alpha-2 \delta}\right) \gamma_{j}^{\frac{1}{\alpha-2 \delta}}\right) & =\exp \left(\sum_{j=1}^{s} \log \left(1+\mu\left(\frac{\alpha}{\alpha-2 \delta}\right) \gamma_{j}^{\frac{1}{\alpha-2 \delta}}\right)\right) \\
& \leq \exp \left(\mu\left(\frac{\alpha}{\alpha-2 \delta}\right) \sum_{j=1}^{s} \gamma_{j}^{\frac{1}{\alpha-2 \delta}}\right),
\end{aligned}
$$

where we used the fact that $\log (1+x) \leq x$ for all $x \geq 0$. Therefore $c_{\infty, \alpha, \gamma, \delta}<\infty$ provided that (4.9) is satisfied. Obviously $c_{s, \alpha, \gamma, \delta} \leq c_{\infty, \alpha, \gamma, \delta}$ and so the second part follows.

For the third part of the theorem observe that $A<\infty$ and therefore for any positive $\eta$ there exists a positive $s_{\eta}$ such that

$$
\sum_{j=1}^{s} \gamma_{j} \leq(A+\eta) \log s \quad \text { for all } s \geq s_{\eta} .
$$

Hence

$$
\begin{aligned}
c_{s, \alpha, \boldsymbol{\gamma},(\alpha-1) / 2}^{2} & =2 \prod_{j=1}^{s}\left(1+\gamma_{j} \mu(\alpha)\right)=2 s^{\sum_{j=1}^{s} \frac{\log \left(1+\gamma_{j} \mu(\alpha)\right)}{\log s}} \\
& \leq 2 s^{\mu(\alpha) \sum_{j=1}^{s} \gamma_{j} / \log s} \leq 2 s^{\mu(\alpha)(A+\eta)},
\end{aligned}
$$

for any $\eta>0$ and all $s \geq s_{\eta}$. Thus there is a constant $\widetilde{c}_{\eta}$ such that

$$
c_{s, \alpha, \gamma,(\alpha-1) / 2} \leq \widetilde{c}_{\eta} s^{\mu(\alpha)(A+\eta) / 2}
$$

and the result follows.

4.3. A Korobov type construction of polynomial lattice rules for integration in $H_{\mathrm{wal}, b, s, \alpha, \gamma}$. In the method of good lattice points one often restricts attention to lattice points whose coordinates are successive powers of a single integer. Such a choice was first proposed by Korobov (see [11]), and therefore such lattice points are often called Korobov lattice points. Here we consider now $s$-tuples $\boldsymbol{q}=\left(q_{1}, \ldots, q_{s}\right)$ of polynomials that are obtained by taking a polynomial $q \in R_{b, m}$ and putting $q_{j} \equiv q^{j-1}(\bmod p)$ with $\operatorname{deg} q_{j}<m$, for $1 \leq j \leq s$. For such $s$-tuples we use the notation $\boldsymbol{v}_{s}(q) \equiv\left(1, q, q^{2}, \ldots, q^{s-1}\right)(\bmod p)$. Note that such a construction was previously considered in [14. 
Algorithm 4.6. Given a prime number $b$, a dimension $s \geq 2$, an integer $m \geq 1$ and weights $\gamma=\left(\gamma_{j}\right)_{j \geq 1}$ :

(1) Choose an irreducible polynomial $p \in \mathbb{Z}_{b}[x]$ with $\operatorname{deg}(p)=m$.

(2) Find $\tilde{q} \in R_{b, m}$ by minimizing $e_{b^{m}, s}^{2}\left(\boldsymbol{v}_{s}(q), p\right)$.

We have the following error bound.

Theorem 4.7. Let $b$ be prime, $s \geq 2$ and let $p \in \mathbb{Z}_{b}[x]$ be irreducible with $\operatorname{deg}(p)=$ $m \geq 1$. A minimizer $\tilde{q}$ obtained from Algorithm 4.6 satisfies

$$
e_{b^{m}, s}^{2}\left(\boldsymbol{v}_{s}(\tilde{q}), p\right) \leq \frac{s}{b^{m}-1} \prod_{j=1}^{s}\left(1+\gamma_{j} \mu(\alpha)\right) \text {. }
$$

Proof. Let $\tilde{q}$ be a minimizer of $e_{b^{m}, s}^{2}\left(\boldsymbol{v}_{s}(q), p\right)$. We are interested in how small $e_{b^{m}, s}^{2}\left(\boldsymbol{v}_{s}(\tilde{q}), p\right)$ is. To this end we define

$$
M_{s}(p):=\frac{1}{b^{m}-1} \sum_{q \in R_{b, m}} e_{b^{m}, s}^{2}\left(\boldsymbol{v}_{s}(q), p\right) .
$$

From Lemma 4.1 we obtain

$$
\begin{aligned}
M_{s}(p) & =\frac{1}{b^{m}-1} \sum_{q \in R_{b, m}} \sum_{\boldsymbol{k} \in D} r_{b}(\alpha, \boldsymbol{\gamma}, \boldsymbol{k}) \\
& =\frac{1}{b^{m}-1} \sum_{\boldsymbol{k} \in \mathbb{N}_{0}^{s} \backslash\{\mathbf{0}\}} r_{b}(\alpha, \gamma, \boldsymbol{k}) \sum_{\substack{q \in R_{b, m} \\
\operatorname{tr}_{m}(\boldsymbol{k}) \cdot \boldsymbol{v}_{s}(q)=0(\bmod p)}} 1 .
\end{aligned}
$$

Now we recall that for an irreducible polynomial $p \in \mathbb{Z}_{b}[x]$ with $\operatorname{deg}(p)=m \geq 1$ and a nonzero $\left(k_{1}, \ldots, k_{s}\right) \in \mathbb{Z}_{b}[x]^{s}$ with $\operatorname{deg}\left(k_{j}\right)<m, j=1, \ldots, s$, the congruence

$$
k_{1}+k_{2} q+\cdots+k_{s} q^{s-1} \equiv 0(\bmod p)
$$

has no solution if $k_{2}=\cdots=k_{s}=0$, and it has at most $s-1$ solutions $q \in R_{b, m}$ otherwise.

For $\boldsymbol{k}=\left(k_{1}, \ldots, k_{s}\right) \in \mathbb{N}_{0}^{s}, \boldsymbol{k} \neq \mathbf{0}$, we consider two cases:

(1) For $j=2, \ldots, s$ let $k_{j}=b^{m} l_{j}, l_{j} \geq 0$. In this case we have $\operatorname{tr}_{m}\left(k_{j}\right)=0$ for $2 \leq j \leq s$ and therefore

$$
\sum_{\substack{q \in R_{b, m} \\ \operatorname{tr}_{m}(\boldsymbol{k}) \cdot v_{s}(q) \equiv 0(\bmod p)}} 1=0
$$

(2) For $j=2, \ldots, s$ let $k_{j}=k_{j}^{*}+b^{m} l_{j}, l_{j} \geq 0,0 \leq k_{j}^{*} \leq b^{m}-1$ and $\left(k_{2}^{*}, \ldots, k_{s}^{*}\right) \neq(0, \ldots, 0)$. Then we obtain

$$
\sum_{\substack{q \in R_{b, m} \\ \operatorname{tr}_{m}(\boldsymbol{k}) \cdot \boldsymbol{v}_{s}(q) \equiv 0(\bmod p)}} 1 \leq s-1 .
$$


Now we have

$$
\begin{aligned}
M_{s}(p) \leq & \sum_{\boldsymbol{k} \in \mathbb{N}_{0}^{s} \backslash\{0\}} r_{b}\left(\alpha, \gamma, b^{m}, \boldsymbol{k}\right)+\frac{s-1}{b^{m}-1} \\
& \times\left[\sum_{k_{1}=0}^{\infty} \sum_{l_{2}, \ldots, l_{s}=0}^{\infty} \sum_{\substack{\left.k_{2}^{*}, \ldots, k_{2}^{*}, \ldots, \ldots, k_{s}^{*}\right) \neq(0, \ldots, 0) \\
b^{m}-1}}^{\infty} r_{b}\left(\alpha, \gamma_{1}, k_{1}\right) \prod_{j=2}^{s} r_{b}\left(\alpha, \gamma_{j}, k_{j}^{*}+l_{j} b^{m}\right)\right] \\
= & \prod_{j=1}^{s}\left(1+\gamma_{j} \frac{\mu(\alpha)}{b^{m \alpha}}\right)-1+\frac{s-1}{b^{m}-1}\left(1+\gamma_{1} \mu(\alpha)\right) \\
& \times\left[\sum_{k_{2}, \ldots, k_{s}=0}^{\infty} \prod_{j=2}^{s} r_{b}\left(\alpha, \gamma_{j}, k_{j}\right)-\sum_{l_{2}, \ldots, l_{s}=0}^{\infty} \prod_{j=2}^{s} r_{b}\left(\alpha, \gamma_{j}, l_{j} b^{m}\right)\right] \\
= & \frac{1}{b^{m \alpha}} \prod_{j=1}^{s}\left(1+\gamma_{j} \mu(\alpha)\right)+\frac{s-1}{b^{m}-1}\left(1+\gamma_{1} \mu(\alpha)\right) \\
& \times\left[\prod_{j=2}^{s}\left(1+\gamma_{j} \mu(\alpha)\right)-\prod_{j=2}^{s}\left(1+\gamma_{j} \frac{\mu(\alpha)}{b^{m \alpha}}\right)\right]
\end{aligned}
$$

and therefore

$$
M_{s}(p) \leq \frac{s}{b^{m}-1} \prod_{j=1}^{s}\left(1+\gamma_{j} \mu(\alpha)\right) .
$$

But since $e_{b^{m}, s}^{2}\left(\boldsymbol{v}_{s}(\tilde{q}), p\right)$ is no larger than the average $M_{s}(p)$, the result follows.

Corollary 4.8. Let $b$ be prime, $s \geq 2, p \in \mathbb{Z}_{b}[x]$ be irreducible with $\operatorname{deg}(p)=m \geq 1$ and $n=b^{m}$. Suppose $\tilde{q} \in R_{b, m}$ is constructed by Algorithm 4.6 .

(1) We have

$$
e_{n, s}\left(\boldsymbol{v}_{s}(\tilde{q}), p\right) \leq c_{s, \alpha, \boldsymbol{\gamma}, \delta} s^{\frac{\alpha}{2}-\delta} n^{-\frac{\alpha}{2}+\delta} \quad \text { for all } 0<\delta \leq \frac{\alpha-1}{2},
$$

where

$$
c_{s, \alpha, \boldsymbol{\gamma}, \delta}:=2^{\frac{\alpha}{2}-\delta} \prod_{j=1}^{s}\left(1+\gamma_{j}^{\frac{1}{\alpha-2 \delta}} \mu\left(\frac{\alpha}{\alpha-2 \delta}\right)\right)^{\frac{\alpha}{2}-\delta} .
$$

(2) Under the assumption

$$
A:=\limsup _{s \rightarrow \infty} \frac{\sum_{j=1}^{s} \gamma_{j}}{\log s}<\infty
$$

we obtain $c_{s, \alpha, \gamma,(\alpha-1) / 2} \leq \widetilde{c}_{\eta} s^{\mu(\alpha)(A+\eta) / 2}$ and therefore

$$
e_{n, s}\left(\boldsymbol{v}_{s}(\tilde{q}), p\right) \leq \widetilde{c}_{\eta} s^{(1+\mu(\alpha)(A+\eta)) / 2} n^{-\frac{1}{2}} \quad \text { for all } \eta>0,
$$

where the constant $\widetilde{c}_{\eta}$ depends only on $\eta$. Thus the worst-case error satisfies a bound which depends only polynomially on the dimension.

Proof. Within this proof we denote the worst-case error $e_{n, s}^{2}\left(\boldsymbol{v}_{s}(q), p\right)$ by $e_{n, s}^{2}\left(\alpha, \gamma, \boldsymbol{v}_{s}(q)\right)$ to stress the dependence on $\alpha$ and $\boldsymbol{\gamma}$.

By Jensen's inequality we obtain

$$
e_{n, s}^{2}\left(\alpha, \gamma, \boldsymbol{v}_{s}(q)\right) \leq\left[e_{n, s}^{2}\left(\alpha \lambda, \gamma^{\lambda}, \boldsymbol{v}_{s}(q)\right)\right]^{1 / \lambda} \quad \text { for all } 1 / \alpha<\lambda \leq 1 .
$$


From Theorem 4.7 we find that there exists a polynomial $q_{*} \in R_{b, m}$ such that

$$
e_{n, s}^{2}\left(\alpha \lambda, \gamma^{\lambda}, \boldsymbol{v}_{s}\left(q_{*}\right)\right) \leq \frac{s}{n-1} \prod_{j=1}^{s}\left(1+\gamma_{j}^{\lambda} \mu(\alpha \lambda)\right) \leq \frac{2 s}{n} \prod_{j=1}^{s}\left(1+\gamma_{j}^{\lambda} \mu(\alpha \lambda)\right) .
$$

Therefore for the minimizer $\tilde{q}$ we obtain

$$
e_{n, s}\left(\alpha, \boldsymbol{\gamma}, \boldsymbol{v}_{s}(\tilde{q})\right) \leq \frac{(2 s)^{\frac{1}{2 \lambda}}}{n^{\frac{1}{2 \lambda}}} \prod_{j=1}^{s}\left(1+\gamma_{j}^{\lambda} \mu(\alpha \lambda)\right)^{\frac{1}{2 \lambda}} .
$$

Now set $\lambda=1 /(\alpha-2 \delta)$ with $0<\delta \leq(\alpha-1) / 2$ and the first result follows. The second part can be shown as in the proof of Corollary 4.5 .

Note that the first bound in Corollary 4.8 differs from the corresponding bound in Corollary 4.5 only by the additional factor $s^{\frac{\alpha}{2}-\delta}$. Thus, just as in the lattice rule case, the Korobov construction is not as good, in principle, as the component-bycomponent construction.

\section{Multivariate integration in Weighted Sobolev SPaCes}

In this section we develop the theory of the previous chapter also for the integration problem in weighted Sobolev spaces. We use randomly digitally shifted polynomial lattice rules.

5.1. Weighted Sobolev spaces. In this section we introduce the weighted Sobolev space $H_{\mathrm{sob}, s, \boldsymbol{w}, \boldsymbol{\gamma}}$ with reproducing kernel given by (see [4, 12, 30, 31])

$$
K_{\mathrm{sob}, s, \boldsymbol{w}, \boldsymbol{\gamma}}(\boldsymbol{x}, \boldsymbol{y})=\prod_{j=1}^{s}\left(1+\gamma_{j} \varrho_{w_{j}}\left(x_{j}, y_{j}\right)\right),
$$

where $\boldsymbol{w}=\left(w_{1}, \ldots, w_{s}\right) \in[0,1]^{s}$ and

$$
\begin{aligned}
\varrho_{w}(x, y) & =\frac{|x-w|+|y-w|-|x-y|}{2} \\
& = \begin{cases}\min (|x-w|,|y-w|), & \text { if }(x-w)(y-w) \geq 0, \\
0, & \text { otherwise. }\end{cases}
\end{aligned}
$$

The inner product in $H_{\mathrm{sob}, s, \boldsymbol{w}, \boldsymbol{\gamma}}$ is given by

$$
\begin{aligned}
& \langle f, g\rangle_{H_{\mathrm{sob}, s, \boldsymbol{w}, \boldsymbol{\gamma}}} \\
& :=f(\boldsymbol{w}) g(\boldsymbol{w})+\sum_{\substack{u \subseteq\{1, \ldots, s\} \\
u \neq \emptyset}} \prod_{j \in u} \gamma_{j}^{-1} \int_{[0,1)^{|u|}} \frac{\partial^{|u|} f}{\partial \boldsymbol{x}_{u}}\left(\boldsymbol{x}_{u}, \boldsymbol{w}\right) \frac{\partial^{|u|} g}{\partial \boldsymbol{x}_{u}}\left(\boldsymbol{x}_{u}, \boldsymbol{w}\right) \mathrm{d} \boldsymbol{x}_{u},
\end{aligned}
$$

where for $\boldsymbol{x}=\left(x_{1}, \ldots, x_{s}\right)$ and $u \subseteq\{1, \ldots, s\}, u \neq \emptyset$, we use the notation $\boldsymbol{x}_{u}=$ $\left(x_{j}\right)_{j \in u}$ and $\left(\boldsymbol{x}_{u}, \boldsymbol{w}\right)$ denotes the $s$-dimensional vector whose $j$-th component is $x_{j}$ if $j \in u$ and $w_{j}$ if $j \notin u$.

Choose a base $b \geq 2$ and let $x=\frac{x_{1}}{b}+\frac{x_{2}}{b^{2}}+\cdots$ and $\sigma=\frac{\sigma_{1}}{b}+\frac{\sigma_{2}}{b^{2}}+\cdots$ be the base $b$ representation of $x$ and $\sigma$. Then the digitally shifted point $y=x \oplus \sigma$ is given by $y=\frac{y_{1}}{b}+\frac{y_{2}}{b^{2}}+\cdots$, where $y_{i}=x_{i}+\sigma_{i} \in \mathbb{Z}_{b}$. For vectors $\boldsymbol{x}$ and $\boldsymbol{\sigma}$ we define the digitally shifted point $\boldsymbol{x} \oplus \boldsymbol{\sigma}$ componentwise. Obviously, the shift depends on the base $b$. 
Dick and Pillichshammer [5] introduced a 'digital shift invariant kernel' associated with a reproducing kernel. For an arbitrary reproducing kernel $K$ the associated digital shift invariant kernel $K_{\mathrm{ds}, b}$ in base $b$ is defined by (see [5])

$$
K_{\mathrm{ds}, b}(\boldsymbol{x}, \boldsymbol{y}):=\int_{[0,1)^{s}} K(\boldsymbol{x} \oplus \boldsymbol{\sigma}, \boldsymbol{y} \oplus \boldsymbol{\sigma}) \mathrm{d} \boldsymbol{\sigma},
$$

where the shift is in base $b$. They also showed that the digital shift invariant kernel in base $b$ of the weighted Sobolev space $H_{\mathrm{sob}, s, \boldsymbol{w}, \boldsymbol{\gamma}}$ is given by

$$
\begin{aligned}
K_{\mathrm{ds}, b, \boldsymbol{w}, \boldsymbol{\gamma}}(\boldsymbol{x}, \boldsymbol{y}) & =\prod_{j=1}^{s}\left(\sum_{k=0}^{\infty} \hat{r}_{b}\left(w_{j}, \gamma_{j}, k\right)_{b} \mathrm{wal}_{k}\left(x_{j}\right) \overline{b_{b} \mathrm{wal}_{k}\left(y_{j}\right)}\right) \\
& =\sum_{\boldsymbol{k} \in \mathbb{N}_{0}^{s}} \hat{r}_{b}(\boldsymbol{w}, \boldsymbol{\gamma}, \boldsymbol{k})_{b}{ }_{b} \operatorname{wal}_{\boldsymbol{k}}(\boldsymbol{x}) \overline{{ }_{b} \operatorname{wal}_{\boldsymbol{k}}(\boldsymbol{y})},
\end{aligned}
$$

with $\boldsymbol{w}=\left(w_{1}, \ldots, w_{s}\right) \in[0,1]^{s}$ and $\hat{r}_{b}(\boldsymbol{w}, \boldsymbol{\gamma}, \boldsymbol{k})=\prod_{j=1}^{s} \hat{r}_{b}\left(w_{j}, \gamma_{j}, k_{j}\right)$, where

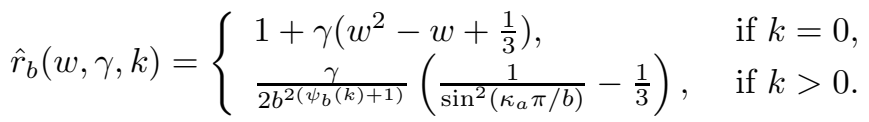

Recall that for $k>0$ we use the notation $k=\kappa_{0}+\kappa_{1} b+\cdots+\kappa_{a} b^{a}$ with $\kappa_{a} \neq 0$ and $\psi_{b}(k)=a$. Furthermore, for $x=\frac{x_{1}}{b}+\frac{x_{2}}{b^{2}}+\cdots$ and $y=\frac{y_{1}}{b}+\frac{y_{2}}{b^{2}}+\cdots$ we define

$$
\begin{aligned}
& \phi_{\mathrm{ds}, b, w}(x, y) \\
& = \begin{cases}w^{2}-w+\frac{1}{2}, & \text { if } x=y, \\
w^{2}-w+\frac{1}{2}-\frac{\left|x_{i_{0}}-y_{i_{0}}\right|\left(b-\left|x_{i_{0}}-y_{i_{0}}\right|\right)}{b^{i_{0}+1},}, & \text { if } x_{1}=y_{1}, \ldots, x_{i_{0}-1}=y_{i_{0}-1}, \\
& \text { and } x_{i_{0}} \neq y_{i_{0}} .\end{cases}
\end{aligned}
$$

Then the shift invariant kernel $K_{\mathrm{ds}, b, \boldsymbol{w}, \boldsymbol{\gamma}}(\boldsymbol{x}, \boldsymbol{y})$ can be rewritten as (see [5, Subsection 6.2])

$$
K_{\mathrm{ds}, b, \boldsymbol{w}, \boldsymbol{\gamma}}(\boldsymbol{x}, \boldsymbol{y})=\prod_{j=1}^{s}\left(1+\gamma_{j} \phi_{\mathrm{ds}, b, w_{j}}\left(x_{j}, y_{j}\right)\right) .
$$

For a point set $P_{n}=\left\{\boldsymbol{x}_{0}, \ldots, \boldsymbol{x}_{n-1}\right\}$ and a $\boldsymbol{\sigma} \in[0,1)^{s}$ let $P_{n, \boldsymbol{\sigma}}=\left\{\boldsymbol{x}_{0} \oplus\right.$ $\left.\boldsymbol{\sigma}, \ldots, \boldsymbol{x}_{n-1} \oplus \boldsymbol{\sigma}\right\}$ be the digitally shifted point set. Furthermore, we recall that for a reproducing kernel Hilbert space $H$ with reproducing kernel $K$ and a point set $P_{n}$ the worst-case error $e\left(P_{n}, K\right)$ is defined as

$$
e\left(P_{n}, K\right):=\sup _{f \in H,\|f\| \leq 1}\left|I(f)-Q_{n, s}\left(P_{n}, f\right)\right| .
$$

Let the mean square worst-case error $\hat{e}^{2}\left(P_{n}, K\right)$ be given by

$$
\hat{e}^{2}\left(P_{n}, K\right):=\mathbb{E}\left[e^{2}\left(P_{n, \boldsymbol{\sigma}}, K\right)\right]=\int_{[0,1)^{s}} e^{2}\left(P_{n, \boldsymbol{\sigma}}, K\right) \mathrm{d} \boldsymbol{\sigma} .
$$

Then we have

$$
\hat{e}^{2}\left(P_{n}, K\right)=e^{2}\left(P_{n}, K_{\mathrm{ds}}\right),
$$

as shown in [5, Theorem 7]. Now with the same arguments as in [5, Theorem 8] we can show that the mean square worst-case error $\hat{e}^{2}\left(P_{n}, K_{\mathrm{sob}, s, \boldsymbol{w}, \gamma}\right)$ for multivariate integration in the weighted Sobolev space $H_{\mathrm{sob}, s, \boldsymbol{w}, \gamma}$ by using a random digital shift 
in base $b$ on the point set $P_{n}=\left\{\boldsymbol{x}_{0}, \ldots, \boldsymbol{x}_{n-1}\right\}$, with $\boldsymbol{x}_{h}=\left(x_{h, 1}, \ldots, x_{h, s}\right)$, is given by

$$
\begin{aligned}
\hat{e}^{2}\left(P_{n}, K_{\mathrm{sob}, s, \boldsymbol{w}, \boldsymbol{\gamma}}\right)= & -\prod_{j=1}^{s}\left(1+\gamma_{j}\left(w_{j}^{2}-w_{j}+\frac{1}{3}\right)\right) \\
& +\frac{1}{n^{2}} \sum_{h, i=0}^{n-1} \sum_{\boldsymbol{k} \in \mathbb{N}_{0}^{s}} \hat{r}_{b}(\boldsymbol{w}, \boldsymbol{\gamma}, \boldsymbol{k})_{b} \mathrm{wal}_{\boldsymbol{k}}\left(\boldsymbol{x}_{h}\right) \overline{b^{\mathrm{wal}} \boldsymbol{w a l}_{\boldsymbol{k}}\left(\boldsymbol{x}_{i}\right)} \\
= & -\prod_{j=1}^{s}\left(1+\gamma_{j}\left(w_{j}^{2}-w_{j}+\frac{1}{3}\right)\right) \\
& +\frac{1}{n^{2}} \sum_{h, i=0}^{n-1} \prod_{j=1}^{s}\left(1+\gamma_{j} \phi_{\mathrm{ds}, b, w_{j}}\left(x_{h, j}, x_{i, j}\right)\right),
\end{aligned}
$$

where the function $\phi_{\mathrm{ds}, b, w}$ is given by (5.2). For the special case where the point set $P$ is a digital $(t, m, s)$-net over $\mathbb{Z}_{b}$ with generating matrices $C_{1}, \ldots, C_{s}$, the mean square worst-case error can be written as

$$
\hat{e}^{2}\left(P, K_{\mathrm{sob}, s, \boldsymbol{w}, \gamma}\right)=\sum_{\boldsymbol{k} \in D} \hat{r}_{b}(\boldsymbol{w}, \boldsymbol{\gamma}, \boldsymbol{k})
$$

where

$$
D=\left\{\boldsymbol{k} \in \mathbb{N}_{0}^{s} \backslash\{\mathbf{0}\}: C_{1}^{T} \operatorname{tr}_{m}\left(\vec{k}_{1}\right)+\cdots+C_{s}^{T} \operatorname{tr}_{m}\left(\vec{k}_{s}\right)=\overrightarrow{0}\right\} .
$$

Furthermore, we have

$$
\begin{aligned}
\hat{e}^{2}\left(P, K_{\mathrm{sob}, s, \boldsymbol{w}, \gamma}\right)= & -\prod_{j=1}^{s}\left(1+\gamma_{j}\left(w_{j}^{2}-w_{j}+\frac{1}{3}\right)\right) \\
& +\frac{1}{n} \sum_{h=0}^{b^{m}-1} \prod_{j=1}^{s}\left(1+\gamma_{j} \phi_{\mathrm{ds}, b, w_{j}}\left(x_{h, j}, 0\right)\right),
\end{aligned}
$$

where $\phi_{\mathrm{ds}, b, w}$ is given by (5.2). For a proof of those results see [5].

As can be seen from (5.2), the function values of $\phi_{\mathrm{ds}, b, w}$ can be computed easily for any $x$ and $y$ and therefore $\hat{e}^{2}\left(P, K_{\mathrm{sob}, s, \boldsymbol{w}, \boldsymbol{\gamma}}\right)$ can be computed in $O\left(b^{m} s\right)$ operations for a given digital net $P$. We will use this fact in the following subsection to search for good digital nets.

Note that, as opposed to the previous section, the base $b$ is not part of the definition of the space, but part of the randomization method. Therefore the base $b$ can be chosen arbitrarily, whereas in the previous section the $b$ was determined by the space $H_{\mathrm{wal}, b, s, \alpha, \boldsymbol{\gamma}}$.

5.2. The component-by-component construction of polynomial lattice rules for integration in weighted Sobolev spaces. In this section we consider digital nets based on Niederreiter's construction using polynomials. We have the following lemma.

Lemma 5.1. Let $p \in \mathbb{Z}_{b}[x]$ with $\operatorname{deg}(p)=m \geq 1$ and let $\boldsymbol{q}=\left(q_{1}, \ldots, q_{s}\right) \in \mathbb{Z}_{b}[x]^{s}$. Then the mean square worst-case error $\hat{e}_{b^{m}, s}^{2}(\boldsymbol{q}, p):=\hat{e}_{b^{m}, s}^{2}\left(S_{p}(\boldsymbol{q}), H_{\mathrm{sob}, s, \boldsymbol{w}, \gamma}\right)$ for integration in the Sobolev space $H_{\mathrm{sob}, s, \boldsymbol{w}, \boldsymbol{\gamma}}$ is given by

$$
\hat{e}_{b^{m}, s}^{2}(\boldsymbol{q}, p)=\sum_{\boldsymbol{k} \in D} \hat{r}_{b}(\boldsymbol{w}, \boldsymbol{\gamma}, \boldsymbol{k})
$$


where

$$
D=\left\{\boldsymbol{k} \in \mathbb{N}_{0}^{s} \backslash\{\mathbf{0}\}: \operatorname{tr}_{m}(\boldsymbol{k}) \cdot \boldsymbol{q} \equiv 0(\bmod p)\right\} .
$$

Proof. The result follows from (5.4) together with (4.3).

Using the above lemma, suitable polynomials can be found using the following algorithm.

Algorithm 5.2. Given a dimension $s$, an integer $m \geq 1$ and weights $\gamma=\left(\gamma_{j}\right)_{j \geq 1}$ :

(1) Choose a prime number $b$ and an irreducible polynomial $p \in \mathbb{Z}_{b}[x]$ with $\operatorname{deg}(p)=m$.

(2) Set $q_{1}=1$.

(3) For $d=2,3, \ldots, s$, find $q_{d} \in R_{b, m}$ by minimizing the square worst-case error $\hat{e}_{b^{m}, d}^{2}\left(\left(q_{1}, \ldots, q_{d}\right), p\right)$.

As for the space $H_{\mathrm{wal}, b, s, \alpha, \gamma}$ we also obtain the following bound.

Theorem 5.3. Let $b$ be prime and let $p \in \mathbb{Z}_{b}[x]$ be irreducible, with $\operatorname{deg}(p)=$ $m \geq 1$. Suppose $\left(q_{1}^{*}, \ldots, q_{s}^{*}\right) \in R_{b, m}^{s}$ is constructed by Algorithm 5.2 . Then for all $d=1,2, \ldots, s$ we have

$$
\begin{aligned}
& \hat{e}_{b^{m}, d}^{2}\left(\left(q_{1}^{*}, \ldots, q_{d}^{*}\right), p\right) \\
& \quad \leq\left(b^{m}-1\right)^{-\frac{1}{\lambda}} \prod_{j=1}^{d}\left(\left(1+\gamma_{j}\left[w_{j}^{2}-w_{j}+\frac{1}{3}\right]\right)^{\lambda}+\tau_{b}(\lambda) \gamma_{j}^{\lambda}\right)^{\frac{1}{\lambda}}
\end{aligned}
$$

for all $\frac{1}{2}<\lambda \leq 1$. Here, for $\lambda=1, \tau_{b}(1):=\frac{1}{6}$ and for $\frac{1}{2}<\lambda<1$ we define

$$
\tau_{2}(\lambda):=\frac{1}{3^{\lambda}\left(2^{2 \lambda}-2\right)} \quad \text { and } \quad \tau_{b}(\lambda):=\frac{\left(4 b^{2}-9\right)^{\lambda}}{54^{\lambda}} \frac{b-1}{\left(b^{2 \lambda}-b\right)} \quad \text { for } b>2 .
$$

Proof. The proof follows exactly the lines of the proof of Theorem 4.4. We just note the following equalities: for any $b \geq 2$ we have

$$
\begin{aligned}
\sum_{k=1}^{\infty} \hat{r}_{b}(w, \gamma, k) & =\sum_{a=0}^{\infty} \sum_{k=b^{a}}^{b^{a+1}-1} \hat{r}_{b}(w, \gamma, k) \\
& =\sum_{a=0}^{\infty} \frac{\gamma b^{a+1}}{2 b^{2 a+2}} \sum_{\kappa_{a}=1}^{b-1}\left(\frac{1}{\sin ^{2}\left(\kappa_{a} \pi / b\right)}-\frac{1}{3}\right) .
\end{aligned}
$$

We have

$$
\sum_{a=1}^{\infty} b^{-a}=\frac{1}{b-1} \quad \text { and } \quad \sum_{\kappa_{a}=1}^{b-1} \frac{1}{\sin ^{2}\left(\kappa_{a} \pi / b\right)}=\frac{b^{2}-1}{3},
$$

where the second equality is shown in [5, Appendix C]. Thus we obtain

$$
\sum_{k=1}^{\infty} \hat{r}_{b}(w, \gamma, k)=\frac{\gamma}{2 b(b-1)}\left(\frac{b^{2}-1}{3}-\frac{b-1}{3}\right)=\frac{\gamma}{6}=\gamma \tau_{b}(1) .
$$


Furthermore, we have $\hat{r}_{2}(w, \gamma, k)=\frac{\gamma}{3 \cdot 2^{2 a+2}}$ for $k>0$ and therefore

$$
\begin{aligned}
\sum_{k=1}^{\infty} \hat{r}_{2}(w, \gamma, k)^{\lambda} & =\sum_{a=0}^{\infty} \sum_{k=2^{a}}^{2^{a+1}-1} \hat{r}_{2}(w, \gamma, k)^{\lambda}=\sum_{a=0}^{\infty} \frac{\gamma^{\lambda} 2^{a}}{3^{\lambda} 2^{2(a+1) \lambda}} \\
& =\frac{\gamma^{\lambda}}{2 \cdot 3^{\lambda}} \sum_{a=0}^{\infty} \frac{1}{2^{(a+1)(2 \lambda-1)}}=\frac{\gamma^{\lambda}}{3^{\lambda}\left(2^{2 \lambda}-2\right)}=\gamma^{\lambda} \tau_{2}(\lambda)
\end{aligned}
$$

for any $1 / 2<\lambda \leq 1$. For $b>2$ we estimate $\sin \left(\kappa_{a} \pi / b\right) \geq \sin (\pi / b) \geq \frac{3 \sqrt{3}}{2 b}$ and therefore

$$
\frac{1}{\sin ^{2}\left(\kappa_{a} \pi / b\right)}-\frac{1}{3} \leq \frac{4 b^{2}-9}{27} .
$$

Using this estimation, we get

$$
\begin{aligned}
\sum_{k=1}^{\infty} \hat{r}_{b}(w, \gamma, k)^{\lambda} & =\sum_{a=0}^{\infty} \sum_{k=b^{a}}^{b^{a+1}-1} \hat{r}_{b}(w, \gamma, k)^{\lambda} \\
& \leq \sum_{a=0}^{\infty} \frac{\gamma^{\lambda} b^{a}(b-1)\left(4 b^{2}-9\right)^{\lambda}}{2^{\lambda} b^{2 \lambda(a+1)} 27^{\lambda}} \\
& =\frac{\gamma^{\lambda}(b-1)\left(4 b^{2}-9\right)^{\lambda}}{54^{\lambda}\left(b^{2 \lambda}-b\right)}=\gamma^{\lambda} \tau_{b}(\lambda),
\end{aligned}
$$

for any $1 / 2<\lambda \leq 1$.

As above (see Corollary 4.5) we obtain the following corollary.

Corollary 5.4. Let $b$ be prime, let $p \in \mathbb{Z}_{b}[x]$ be irreducible with $\operatorname{deg}(p)=m \geq 1$ and let $n=b^{m}$. Suppose $\boldsymbol{q}^{*} \in R_{b, m}^{s}$ is constructed by Algorithm 5.2 ,

(1) We have

$$
\hat{e}_{n, s}\left(\boldsymbol{q}^{*}, p\right) \leq c_{s, \boldsymbol{w}, \boldsymbol{\gamma}, \delta} n^{-1+\delta} \quad \text { for all } 0<\delta \leq \frac{1}{2}
$$

where

$$
\begin{aligned}
& c_{s, \boldsymbol{w}, \boldsymbol{\gamma}, \delta} \\
& \quad:=2^{1-\delta} \prod_{j=1}^{s}\left(1+\gamma_{j}^{\frac{1}{2(1-\delta)}}\left[\left(w_{j}^{2}-w_{j}+\frac{1}{3}\right)^{\frac{1}{2(1-\delta)}}+\tau_{b}\left(\frac{1}{2(1-\delta)}\right)\right]\right)^{1-\delta} .
\end{aligned}
$$

(2) Suppose

$$
\sum_{j=1}^{\infty} \gamma_{j}^{\frac{1}{2(1-\delta)}}<\infty .
$$

Then $c_{s, \boldsymbol{w}, \boldsymbol{\gamma}, \delta} \leq c_{\infty, \boldsymbol{w}, \boldsymbol{\gamma}, \delta}<\infty$ and we have

$$
\hat{e}_{n, s}\left(\boldsymbol{q}^{*}, p\right) \leq c_{\infty, \boldsymbol{w}, \boldsymbol{\gamma}, \delta} n^{-1+\delta} \quad \text { for all } 0<\delta \leq \frac{1}{2} .
$$

Thus the root mean square worst-case error of the point set $S_{p}(\boldsymbol{q})$ is bounded independently of the dimension.

(3) Under the assumption

$$
A:=\limsup _{s \rightarrow \infty} \frac{\sum_{j=1}^{s} \gamma_{j}}{\log s}<\infty
$$


we obtain $c_{s, \boldsymbol{w}, \boldsymbol{\gamma}, 1 / 2} \leq \widetilde{c}_{\eta} s^{(A+\eta) / 2}$ and therefore

$$
\hat{e}_{n, s}\left(\boldsymbol{q}^{*}, p\right) \leq \widetilde{c}_{\eta} s^{(A+\eta) / 2} n^{-\frac{1}{2}} \quad \text { for all } \eta>0,
$$

where the constant $\widetilde{c}_{\eta}$ depends only on $\eta$. Thus the root mean square worstcase error of the point set $S_{p}(\boldsymbol{q})$ satisfies a bound which depends only polynomially on the dimension.

5.3. A Korobov type construction of polynomial lattice rules for integration in weighted Sobolev spaces. We can also obtain results for Korobov type rules. As before we use the notation $\boldsymbol{v}_{s}(q) \equiv\left(1, q, q^{2}, \ldots, q^{s-1}\right)(\bmod p)$. We have the following algorithm.

Algorithm 5.5. Given a dimension $s \geq 2$, an integer $m \geq 1$ and weights $\gamma=$ $\left(\gamma_{j}\right)_{j \geq 1}$ :

(1) Choose a prime number $b$ and an irreducible polynomial $p \in \mathbb{Z}_{b}[x]$ with $\operatorname{deg}(p)=m$

(2) Find $\tilde{q} \in R_{b, m}$ by minimizing $\hat{e}_{b^{m}, s}^{2}\left(\boldsymbol{v}_{s}(q), p\right)$.

The following results can be shown as in Subsection 4.3 .

Theorem 5.6. Let $b$ be prime, $s \geq 2$ and $p \in \mathbb{Z}_{b}[x]$ be irreducible, with $\operatorname{deg}(p)=$ $m \geq 1$. Let $\tau_{b}$ be defined as in Theorem [5.3. Suppose $\tilde{q} \in R_{b, m}$ is constructed by Algorithm 5.5. Then we have

$$
\hat{e}_{b^{m}, s}^{2}\left(\boldsymbol{v}_{s}(\tilde{q}), p\right) \leq\left(\frac{s}{q^{m}-1}\right)^{\frac{1}{\lambda}} \prod_{j=1}^{s}\left(\left(1+\gamma_{j}\left[w_{j}^{2}-w_{j}+\frac{1}{3}\right]\right)^{\lambda}+\tau_{b}(\lambda) \gamma_{j}^{\lambda}\right)^{\frac{1}{\lambda}}
$$

for all $\frac{1}{2}<\lambda \leq 1$.

Corollary 5.7. Let $b$ be prime, let $s \geq 2$, let $p \in \mathbb{Z}_{b}[x]$ be irreducible with $\operatorname{deg}(p)=$ $m \geq 1$ and let $n=b^{m}$. Suppose $\tilde{q} \in R_{b, m}$ is constructed by Algorithm 5.5.

(1) We have

$$
\hat{e}_{n, s}\left(\boldsymbol{v}_{s}(\tilde{q}), p\right) \leq c_{s, \boldsymbol{w}, \boldsymbol{\gamma}, \delta} s^{1-\delta} n^{-1+\delta} \quad \text { for all } 0<\delta \leq \frac{1}{2},
$$

where

$$
\begin{aligned}
& c_{s, \boldsymbol{w}, \boldsymbol{\gamma}, \delta} \\
& :=2^{1-\delta} \prod_{j=1}^{s}\left(1+\gamma_{j}^{\frac{1}{2(1-\delta)}}\left[\left(w_{j}^{2}-w_{j}+\frac{1}{3}\right)^{\frac{1}{2(1-\delta)}}+\tau_{b}\left(\frac{1}{2(1-\delta)}\right)\right]\right)^{1-\delta} .
\end{aligned}
$$

(2) Under the assumption

$$
A:=\limsup _{s \rightarrow \infty} \frac{\sum_{j=1}^{s} \gamma_{j}}{\log s}<\infty
$$

we obtain $\hat{c}_{s, \boldsymbol{w}, \boldsymbol{\gamma}, 1 / 2} \leq \bar{c}_{\eta} s^{(A+\eta) / 2}$ and therefore

$$
\hat{e}_{n, s}\left(\boldsymbol{v}_{s}(\tilde{q}), p\right) \leq \bar{c}_{\eta} s^{(1+(A+\eta)) / 2} n^{-\frac{1}{2}} \quad \text { for all } \eta>0,
$$

where the constant $\bar{c}_{\eta}$ depends only on $\eta$. Thus the root mean square worstcase error of the point set $S_{p}\left(\boldsymbol{v}_{s}(\tilde{q})\right)$ satisfies a bound which depends only polynomially on the dimension. 


\section{NUMERICAL RESULTS}

In this section we present numerical results for the worst-case error of integration in weighted Sobolev spaces. The aim is to compare the performance of polynomial lattice rules with those of lattice rules. As previously done for lattice rules, we choose $w_{j}=1$ for $j=1, \ldots, s$, the dimension $s=100$ and we consider the weights

$$
\gamma_{j}=1, \quad \gamma_{j}=0.5^{j}, \quad \gamma_{j}=j^{-2}, \quad \gamma_{j, s}=\frac{10}{s}=\frac{1}{10} .
$$

Note that we can also allow the weights $\gamma_{j}$ to depend on the dimension $s$, that is, $\gamma_{j}=\gamma_{j, s} ;$ see [6].

The simplest and also most efficient choice for our constructive algorithms is obtained by choosing $b=2$. The mean square worst-case error (see (5.5)) is then given by

$$
\hat{e}^{2}\left(S_{p}(\boldsymbol{q}), K_{\mathrm{sob}, s, \mathbf{1}, \boldsymbol{\gamma}}\right)=-\prod_{j=1}^{s}\left(1+\frac{\gamma_{j}}{3}\right)+\frac{1}{n} \sum_{h=0}^{2^{m}-1} \prod_{j=1}^{s}\left(1+\gamma_{j} \phi_{\mathrm{ds}, 2,1}\left(x_{h, j}, 0\right)\right),
$$

where $($ see $(5.2))$

$$
\phi_{\mathrm{ds}, 2,1}(x, 0)= \begin{cases}\frac{1}{2}, & \text { if } x=0, \\ \frac{1}{2}-2^{\left\lfloor\log _{2} x\right\rfloor-1}, & \text { otherwise, }\end{cases}
$$

where $\log _{2}$ denotes the logarithm in base 2 .

Tables of the worst-case error of polynomial lattice rules (plr) and lattice rules (lr), which are constructed by a component-by-component algorithm (CBC) or by Korobov's construction method (Korobov), are presented in the appendix. (The irreducible polynomials $p \in \mathbb{Z}_{2}[x]$ used in these computations are given in Table 1.)

Furthermore, we compare the worst-case errors obtained from different choices of irreducible polynomials $p \in \mathbb{Z}_{2}[x]$. These results are shown in Tables 6 and 7 . Again we choose $s=100$ and we consider $n=1024$ and 2048 and $\gamma_{j}=j^{-2}$ and $\gamma_{j}=\frac{1}{10}$.

Concluding remarks. The upper bounds on the mean square worst-case error for integration in weighted Sobolev spaces using randomly digitally shifted polynomial lattice rules are almost the same as for randomly shifted lattice rules. Surprisingly enough we obtain exactly the same constant in Theorem 5.3 and Theorem 5.6 by taking $\lambda=1$ as for the corresponding bounds for lattice rules; see [4, 38.

Numerically it appears that polynomial lattice rules constructed by a componentby-component algorithm are slightly better (see Tables 2 to 5) than lattice rules constructed by a component-by-component algorithm. For the Korobov construction sometimes polynomial lattice rules are better, and sometimes lattice rules are better. In any case, the difference is rather small.

Tables 6 and 7 suggest that the choice of the polynomial $p$ has only a small influence on the outcome. This influence seems to be slightly stronger for the Korobov construction. Still it seems that the particular choice of the irreducible polynomial $p$ is of no significance. This is also validated by our theory, which only depends on the degree of the polynomial $p$, but not on the particular choice.

The component-by-component construction of polynomial lattice rules yields a construction extensible in the dimension. In 24] on the other hand it was shown by Niederreiter that there exist good polynomial lattice rules which are extensible in both the modulus and the dimension simultaneously. This raises the question 
of whether the algorithms of this paper can be modified to also allow an extension in the number of points. This is an important and seemingly challenging problem, which is left for future work.

\section{Appendix. TABles of numerical Results}

TABLE 1. Irreducible polynomial $p \in \mathbb{Z}_{2}[x]$

\begin{tabular}{|c|c|}
\hline$m$ & $p$ \\
\hline 8 & $x^{8}+x^{5}+x^{4}+x^{3}+1$ \\
9 & $x^{9}+x^{8}+x^{7}+x^{5}+x^{4}+x^{2}+1$ \\
10 & $x^{10}+x^{7}+x^{3}+x+1$ \\
11 & $x^{11}+x^{10}+x^{8}+x^{6}+x^{4}+x^{2}+1$ \\
12 & $x^{12}+x^{9}+x^{8}+x^{7}+x^{6}+x^{4}+x^{2}+x+1$ \\
\hline
\end{tabular}

TABLE 2. Worst-case error, $\gamma_{j}=1$

\begin{tabular}{|c|c|c|c|c|}
\hline$n$ & CBC plr & CBC lr & Korobov plr & Korobov lr \\
\hline 256 & $3.98437 e+07$ & $3.98456 e+07$ & $3.98443 e+07$ & $3.98456 e+07$ \\
512 & $2.81719 e+07$ & $2.81721 e+07$ & $2.81721 e+07$ & $2.81721 e+07$ \\
1024 & $1.99186 e+07$ & $1.99194 e+07$ & $1.99187 e+07$ & $1.99193 e+07$ \\
2048 & $1.40828 e+07$ & $1.40840 e+07$ & $1.40828 e+07$ & $1.40841 e+07$ \\
4096 & $9.95656 e+06$ & $9.95785 e+06$ & $9.95642 e+06$ & $9.95826 e+06$ \\
\hline
\end{tabular}

TABLE 3. Worst-case error, $\gamma_{j}=0.5^{j}$

\begin{tabular}{|c|c|c|c|c|}
\hline$n$ & CBC plr & CBC lr & Korobov plr & Korobov lr \\
\hline 256 & $2.51805 e-03$ & $2.59907 e-03$ & $2.75401 e-03$ & $2.86635 e-03$ \\
512 & $1.33062 e-03$ & $1.36727 e-03$ & $1.49945 e-03$ & $1.46307 e-03$ \\
1024 & $6.95360 e-04$ & $7.14036 e-04$ & $7.84960 e-04$ & $7.75159 e-04$ \\
2048 & $3.61270 e-04$ & $3.80296 e-04$ & $4.14176 e-04$ & $4.20300 e-04$ \\
4096 & $1.90239 e-04$ & $1.94805 e-04$ & $2.23073 e-04$ & $2.15116 e-04$ \\
\hline
\end{tabular}

TABLE 4. Worst-case error, $\gamma_{j}=j^{-2}$

\begin{tabular}{|c|c|c|c|c|}
\hline$n$ & CBC plr & CBC lr & Korobov plr & Korobov lr \\
\hline 256 & $4.23326 e-03$ & $4.43538 e-03$ & $5.51303 e-03$ & $5.75309 e-03$ \\
512 & $2.30490 e-03$ & $2.40453 e-03$ & $3.03423 e-03$ & $3.02527 e-03$ \\
1024 & $1.23355 e-03$ & $1.28702 e-03$ & $1.75583 e-03$ & $1.75881 e-03$ \\
2048 & $6.68382 e-04$ & $6.94185 e-04$ & $9.31863 e-04$ & $9.45105 e-04$ \\
4096 & $3.62609 e-04$ & $3.74410 e-04$ & $5.48164 e-04$ & $5.47949 e-04$ \\
\hline
\end{tabular}


TABLE 5. Worst-case error, $\gamma_{j}=\frac{1}{10}$

\begin{tabular}{|c|c|c|c|c|}
\hline$n$ & CBC plr & CBC lr & Korobov plr & Korobov lr \\
\hline 256 & $4.23940 e-01$ & $4.64515 e-01$ & $4.23140 e-01$ & $4.63965 e-01$ \\
512 & $2.79683 e-01$ & $2.86692 e-01$ & $2.77131 e-01$ & $2.86441 e-01$ \\
1024 & $1.84695 e-01$ & $1.84526 e-01$ & $1.81462 e-01$ & $1.80904 e-01$ \\
2048 & $1.21283 e-01$ & $1.21268 e-01$ & $1.18402 e-01$ & $1.20839 e-01$ \\
4096 & $8.00544 e-02$ & $8.15918 e-02$ & $7.97847 e-02$ & $8.18734 e-02$ \\
\hline
\end{tabular}

TABLE 6. Worst-case error for various choices of the irreducible polynomial $p$ with $\gamma_{j}=j^{-2}$

\begin{tabular}{|c|c|c|c|}
\hline$n$ & $p$ & CBC plr & Korobov plr \\
\hline \multirow{5}{*}{1024} & $x^{10}+x^{7}+x^{3}+x+1$ & $1.23355 e-03$ & $1.75583 e-03$ \\
& $x^{10}+x^{9}+x^{7}+x^{6}+x^{4}+x^{3}+x^{2}+x+1$ & $1.23383 e-03$ & $1.72422 e-03$ \\
& $x^{10}+x^{9}+x^{8}+x^{7}+x^{6}+x^{4}+x^{3}+x+1$ & $1.22844 e-03$ & $1.78052 e-03$ \\
& $x^{10}+x^{8}+x^{4}+x^{3}+1$ & $1.22893 e-03$ & $1.71933 e-03$ \\
\hline \multirow{4}{*}{2048} & $x^{10}+x^{8}+x^{7}+x^{6}+1$ & $1.23561 e-03$ & $1.68367 e-03$ \\
\hline & $x^{11}+x^{10}+x^{8}+x^{6}+x^{4}+x^{2}+1$ & $6.68382 e-04$ & $9.31863 e-04$ \\
& $x^{11}+x^{2}+1$ & $6.65375 e-04$ & $9.43137 e-04$ \\
& $x^{11}+x^{10}+x^{9}+x^{5}+x^{2}+x+1$ & $6.68968 e-04$ & $9.40263 e-04$ \\
& $x^{11}+x^{10}+x^{8}+x^{6}+1$ & $6.70797 e-04$ & $1.00080 e-03$ \\
& $x^{11}+x^{10}+x^{8}+x^{6}+x^{5}+x^{4}+1$ & $6.63566 e-04$ & $9.41526 e-04$ \\
\hline
\end{tabular}

TABLE 7. Worst-case error for various choices of the irreducible polynomial $p$ with $\gamma_{j}=\frac{1}{10}$

\begin{tabular}{|c|c|c|c|}
\hline$n$ & $p$ & CBC plr & Korobov plr \\
\hline \multirow{5}{*}{1024} & $x^{10}+x^{7}+x^{3}+x+1$ & $1.84695 e-01$ & $1.81462 e-01$ \\
& $x^{10}+x^{9}+x^{7}+x^{6}+x^{4}+x^{3}+x^{2}+x+1$ & $1.83927 e-01$ & $1.77861 e-01$ \\
& $x^{10}+x^{9}+x^{8}+x^{7}+x^{6}+x^{4}+x^{3}+x+1$ & $1.83857 e-01$ & $1.84145 e-01$ \\
& $x^{10}+x^{8}+x^{4}+x^{3}+1$ & $1.84438 e-01$ & $1.84699 e-01$ \\
& $x^{10}+x^{8}+x^{7}+x^{6}+1$ & $1.84385 e-01$ & $1.82713 e-01$ \\
\hline \multirow{3}{*}{2048} & $x^{11}+x^{10}+x^{8}+x^{6}+x^{4}+x^{2}+1$ & $1.21283 e-01$ & $1.18402 e-01$ \\
& $x^{11}+x^{2}+1$ & $1.21869 e-01$ & $1.20605 e-01$ \\
& $x^{11}+x^{10}+x^{9}+x^{5}+x^{2}+x+1$ & $1.21083 e-01$ & $1.19697 e-01$ \\
& $x^{11}+x^{10}+x^{8}+x^{6}+1$ & $1.21290 e-01$ & $1.19272 e-01$ \\
& $x^{11}+x^{10}+x^{8}+x^{6}+x^{5}+x^{4}+1$ & $1.21721 e-01$ & $1.20039 e-01$ \\
\hline
\end{tabular}

\section{ACKNowledGments}

The support of the Australian Research Council under its Center of Excellence Program is gratefully acknowledged. The third author is supported by the Austrian Research Foundation (FWF), Project S 8305 and P17022-N12. 


\section{REFERENCES}

[1] Aronszajn, N.: Theory of reproducing kernels. Trans. Amer. Math. Soc., 68: 337-404, 1950. $\operatorname{MR} 0051437(14: 479 \mathrm{c})$

[2] Chrestenson, H.E.: A class of generalized Walsh functions. Pacific J. Math., 5: 17-31, 1955. MR0068659 (16:920c)

[3] Cranley, R. and Patterson, T.N.L.: Randomization of number theoretic methods for multiple integration. SIAM J. Numer. Anal., 13: 904-914, 1976. MR0494820 (58:13605)

[4] Dick, J.: On the convergence rate of the component-by-component construction of good lattice rules. J. Complexity, 20: 493-522, 2004. MR2068155

[5] Dick, J., Pillichshammer, F.: Multivariate integration in weighted Hilbert spaces based on Walsh functions and weighted Sobolev spaces. J. Complexity, 21: 149-195, 2005.

[6] Dick, J., Sloan, I.H., Wang, X., and Woźniakowski, H.: Liberating the weights. J. Complexity, 20: 593-623, 2004.

[7] Dick, J., Sloan, I.H., Wang, X. and Woźniakowski, H.: Good lattice rules in weighted Korobov spaces with general weights. Submitted.

[8] Faure, H.: Discrépance de suites associées à un système de numération (en dimension $s$ ). Acta Arith., 41: 337-351, 1982. MR0677547 (84m:10050)

[9] Hickernell, F.J.: Lattice rules: how well do they measure up? In: Random and QuasiRandom Point Sets (P. Hellekalek and G. Larcher, eds.), pp. 109-166, Springer Lecture Notes in Statistics 138, 1998. MR1662841 (2000b:65007)

[10] Korobov, N.M.: The approximate computation of multiple integrals. Dokl. Akad. Nauk SSSR, 124: 1207-1210, 1959. (In Russian) MR0104086 (21:2848)

[11] Korobov, N.M.: Properties and calculation of optimal coefficients. Dokl. Akad. Nauk SSSR 132: 1009-1012, 1960. (In Russian) MR0120768 (22:11517)

[12] Kuo, F.Y.: Component-by-component constructions achieve the optimal rate of convergence for multivariate integration in weighted Korobov and Sobolev spaces. J. Complexity, 19: 301-320, 2003. MR.1984116 (2004c:41066)

[13] Larcher, G.: Digital point sets: analysis and application. In: Random and Quasi-Random Point Sets (P. Hellekalek and G. Larcher, eds.), pp. 167-222, Springer Lecture Notes in Statistics 138, 1998. MR1662842 (99m:11085)

[14] Larcher, G., Lauß, A., Niederreiter, H., and Schmid, W.Ch.:Optimal polynomials for $(t, m, s)$ nets and numerical integration of multivariate Walsh series. SIAM J. Numer. Anal., 33: 2239-2253, 1996. MR1427461 (97m:65046)

[15] Larcher, G., Niederreiter, H., and Schmid, W.Ch.: Digital nets and sequences constructed over finite rings and their application to quasi-Monte Carlo integration. Monatsh. Math., 121: 231-253, 1996. MR1383533(97d:11119)

[16] Lemieux, Ch., L'Ecuyer, P.: Randomized polynomial lattice rules for multivariate integration and simulation, SIAM J. Sci. Comput. 24: 1768-1789, 2003. MR.1978160 (2004e:65009)

[17] Lidl, R., Niederreiter, H.: Introduction to Finite Fields and their Applications. Cambridge Univ. Press, Cambridge, 1994. MR1294139 (95f:11098)

[18] Niederdrenk, K.: Die endliche Fourier- und Walshtransformation mit einer Einführung in die Bildverarbeitung. Vieweg, Braunschweig, 1982. MR0735256 (85g:94001)

[19] Niederreiter, H.: Point sets and sequences with small discrepancy. Monatsh. Math., 104: 273-337, 1987. MR0918037 (89c:11120)

[20] Niederreiter, H.: Low-discrepancy point sets obtained by digital constructions over finite fields. Czechoslovak Math. J., 42: 143-166, 1992. MR1152177(93c:11055)

[21] Niederreiter, H.: Random Number Generation and Quasi-Monte Carlo Methods. No. 63 in CBMS-NSF Series in Applied Mathematics. SIAM, Philadelphia, 1992. MR1172997 (93h:65008)

[22] Niederreiter, H.: Finite fields, pseudorandom numbers, and quasirandom points. In: Finite Fields, Coding Theory, and Advances in Communications and Computing (Mullen, G.L., and Shiue, P.J.-S., eds.), pp. 375-394, Dekker, New York, 1992. MR1199844(94a:11121)

[23] Niederreiter, H.: Constructions of $(t, m, s)$-nets. In: Monte Carlo and Quasi-Monte Carlo Methods 1998 (Niederreiter, H., and Spanier, J., eds.), pp. 70-85, Springer, Berlin, 2000. MR1849843 (2002e:65012)

[24] Niederreiter, H.: The existence of good extensible polynomial lattice rules. Monatsh. Math., 139: 295-307, 2003. MR2001711 (2004j:11087) 
[25] Pirsic, G.: Schnell konvergierende Walshreihen über Gruppen. Master's Thesis, University of Salzburg, 1995. (Available at http://www.ricam.oeaw.ac.at/people/page/pirsic/)

[26] Pirsic, G., Schmid, W.Ch.: Calculation of the quality parameter of digital nets and application to their construction. J. Complexity 17: 827-839, 2001. MR1881672 (2002m:65004)

[27] Rivlin, T.J., Saff, E.B.: Joseph L. Walsh Selected Papers. Springer Verlag, New York, 2000. MR1757949 (2001g:01052)

[28] Schmid, W.Ch.: Improvements and extensions of the "Salzburg tables" by using irreducible polynomials. In: Monte Carlo and quasi-Monte Carlo methods 1998 (Niederreiter, H., et al., eds.), pp. 436-447, Springer, Berlin, 2000. MR.1849869

[29] Sloan, I.H. and Joe, S.: Lattice methods for multiple integration. Oxford University Press, Oxford, 1994. MR 1442955 (98a:65026)

[30] Sloan, I.H., Kuo, F.Y., and Joe, S.: Constructing randomly shifted lattice rules in weighted Sobolev spaces. SIAM J. Numer. Anal., 40: 1650-1655, 2002. MR1950616 (2003m:65031)

[31] Sloan, I.H., Kuo, F.Y., and Joe, S.: On the step-by-step construction of quasi-Monte Carlo integration rules that achieve strong tractability error bounds in weighted Sobolev spaces. Math. Comp., 71: 1609-1640, 2002. MR1933047 (2003m:65030)

[32] Sloan, I.H. and Woźniakowski, H.: When are quasi-Monte Carlo algorithms efficient for high dimensional integrals? J. Complexity, 14: 1-33, 1998. MR1617765 (99d:65384)

[33] Sloan, I.H., Woźniakowski, H.: Tractability of multivariate integration for weighted Korobov classes. J. Complexity, 17: 697-721, 2001. MR1881665 (2003g:65030)

[34] Sobol', I.M.: On the distribution of points in a cube and the approximate evaluation of integrals. U.S.S.R. Comput. Maths. Math. Phys., 7: 86-112, 1967. MR0219238 (36:2321)

[35] Walsh, J.L.: A closed set of normal orthogonal functions. Amer. J. Math., 55: 5-24, 1923.

[36] Wang, X. and Sloan, I.H.: Efficient weighted lattice rules with applications to finance. Submitted.

[37] Wang, X. and Sloan, I.H.: Why are high-dimensional finance problems often of low effective dimension? To appear in SIAM J. Sci. Comput., 2005.

[38] Wang, X., Sloan, I.H. and Dick, J.: On Korobov lattice rules in weighted Korobov spaces. SIAM J. Numer. Anal. 42: 1760-1779, 2004.

School of Mathematics, The University of New South Wales, Sydney, New South Wales 2052, Australia

E-mail address: josi@maths.unsw.edu.au

School of Mathematics, The University of New South Wales, Sydney, New South Wales 2052, Australia

E-mail address: fkuo@maths.unsw.edu.au

Institut für Analysis, Universität Linz, Altenbergstrasse 69, A-4040 LinZ, Austria

E-mail address: friedrich.pillichshammer@jku.at

School of Mathematics, The University of New South Wales, Sydney, New South Wales 2052, Australia

E-mail address: sloan@maths.unsw.edu.au 